\title{
Discovery of glycerol phosphate modification on streptococcal rhamnose polysaccharides
}

\author{
Rebecca J. Edgar ${ }^{1,9}$, Vincent P. van Hensbergen 2,11, Alessandro Ruda $\mathbb{1}^{3,11}$, Andrew G. Turner ${ }^{4}$, \\ Pan Deng ${ }^{1}{ }^{5}$, Yoann Le Breton ${ }^{6,10}$, Najib M. El-Sayed (10,7, Ashton T. Belew ${ }^{6}{ }^{6,7}$, Kevin S. Mclver (1)6, \\ Alastair G. McEwan ${ }^{4}$, Andrew J. Morris ${ }^{5}{ }^{5}$, Gérard Lambeau ${ }^{8}{ }^{8}$, Mark J. Walker ${ }^{4}$, Jeffrey S. Rush', \\ Konstantin V. Korotkov ${ }^{1}{ }^{1}$, Göran Widmalm $\mathbb{1}^{3}{ }^{3}$, Nina M. van Sorge $\mathbb{( i D}^{2,12 \star}$ and Natalia Korotkova $\mathbb{D}^{1,12 \star}$
}

Cell wall glycopolymers on the surface of Gram-positive bacteria are fundamental to bacterial physiology and infection biology. Here we identify gacH, a gene in the Streptococcus pyogenes group A carbohydrate (GAC) biosynthetic cluster, in two independent transposon library screens for its ability to confer resistance to zinc and susceptibility to the bactericidal enzyme human group IIA-secreted phospholipase $A_{2}$. Subsequent structural and phylogenetic analysis of the GacH extracellular domain revealed that GacH represents an alternative class of glycerol phosphate transferase. We detected the presence of glycerol phosphate in the GAC, as well as the serotype c carbohydrate from Streptococcus mutans, which depended on the presence of the respective gacH homologs. Finally, nuclear magnetic resonance analysis of GAC confirmed that glycerol phosphate is attached to approximately $25 \%$ of the GAC $\mathbf{N}$-acetylglucosamine side-chains at the $\mathrm{C} 6$ hydroxyl group. This previously unrecognized structural modification impacts host-pathogen interaction and has implications for vaccine design.

G ram-positive bacteria are surrounded by a thick cell wall consisting of a complex network of peptidoglycan with covalently attached glycopolymers that comprise a large family of structurally diverse molecules, including wall teichoic acid, mycobacterial arabinogalactans and capsular polysaccharides. From these, wall teichoic acid is perhaps the most widespread and best-studied molecule. This polyanionic, phosphate-rich glycopolymer is critical for functions including cell division, antibiotic resistance, metal ion homeostasis, phage-mediated horizontal gene transfer and protection of bacteria from host defense peptides and antimicrobial enzymes ${ }^{1,2}$. As such, these structures and their biosynthetic pathways are attractive targets for antibiotic development and vaccine design. Interestingly, many streptococci lack classical wall teichoic acid and instead express glycopolymers that are characterized by the presence of L-rhamnose (Rha) ${ }^{3}$. These structures comprise about $40-60 \%$ of the bacterial cell wall mass, and are historically used for serological grouping of streptococci ${ }^{3}$. The glycopolymers of two human streptococcal pathogens, S. pyogenes or group A Streptococcus (GAS) and S. mutans, are respectively referred to as group A carbohydrate (GAC) and serotype $c$ carbohydrate (SCC). These glycopolymers share a characteristic $(\rightarrow 3) \alpha-$ Rha $(1 \rightarrow 2)$ $\alpha$-Rha $(1 \rightarrow)$ polyrhamnose backbone, but are serologically distinguished based on their specific glycosyl side-chain residues, that is $\mathrm{N}$-acetyl- $\beta$-D-glucosamine (GlcNAc) in $\mathrm{GAC}^{4}$ and $\alpha$-glucose (Glc) in SCC ${ }^{5}$ GAC and SCC play key roles in cell morphology and division $^{6}$, resistance to certain cell wall-targeting antibiotics ${ }^{7}$, biofilm formation $^{8}$ and pathogenesis of GAS and S. mutans ${ }^{9-11}$. Importantly for both pathogens, GAC and SCC have been evaluated as vaccine antigens. Immunization with GAC or SCC induces opsonophagocytic antibodies that enhance killing of GAS and S. mutans, respectively ${ }^{5,12,13}$. In addition, GAC has proven efficacious as a vaccine antigen through active immunization in mice ${ }^{12,13}$.

The GAC and SCC biosynthetic pathways are encoded by 12-gene clusters ${ }^{3,11}$, herein designated as gacABCDEFGHIJKL and sccABCDEFGHMNPQ (Fig. 1a), respectively. The first seven genes in both clusters are conserved in many streptococcal species, and they participate in polyrhamnose backbone synthesis and trans$\operatorname{port}^{14}$. In GAS, gacI, gacJ, gacK and $g a c L$ encode the machinery to generate and add the GlcNAc side-chain to the polyrhamnose backbone ${ }^{11,15}$, whereas the genes required for Glc side-chain generation are not clearly identified in S. mutans. In addition to these streptococcal species, similar gene clusters are present in a wide variety of streptococcal, lactococcal and enterococcal species ${ }^{3}$.

In addition to the polyrhamnose biosynthesis genes, the GAC and SCC biosynthetic clusters contain further conserved genes of unknown function, $g a c H$ and $s c c H$, respectively, which are annotated as putative glycerol phosphate (GroP) transferases. Recently, we employed the Krmit GAS transposon mutant library ${ }^{16}$ and identified $\mathrm{gacI}$ and $\mathrm{gacH}$ as genes that confer bacterial sensitivity to human group IIA-secreted phospholipase A2 (hGIIA) ${ }^{17}$, an important bactericidal protein of the innate immune system against Gram-positive pathogens ${ }^{18}$. Complementary to that study, we now

'Department of Molecular and Cellular Biochemistry, University of Kentucky, Lexington, KY, USA. ${ }^{2}$ Department of Medical Microbiology, University Medical Center Utrecht, Utrecht University, Utrecht, The Netherlands. ${ }^{3}$ Department of Organic Chemistry, Arrhenius Laboratory, Stockholm University, Stockholm, Sweden. ${ }^{4}$ Australian Infectious Diseases Research Centre and School of Chemistry and Molecular Biosciences, The University of Queensland, Brisbane, Australia. ${ }^{5}$ Division of Cardiovascular Medicine and the Gill Heart Institute, University of Kentucky, Lexington, KY, USA. ${ }^{6}$ Department of Cell Biology \& Molecular Genetics and Maryland Pathogen Research Institute, University of Maryland, College Park, MD, USA. ${ }^{7}$ Center for Bioinformatics and Computational Biology, University of Maryland, College Park, MD, USA. ${ }^{8}$ Université Côte d'Azur, Centre National de la Recherche Scientifique, Institut de Pharmacologie Moléculaire et Cellulaire, Valbonne Sophia Antipolis, France. 'Present address: Callaghan Innovation, Gracefield, Lower Hutt, New Zealand. ${ }^{10}$ Present address: Wound Infections Department, Bacterial Diseases Branch, Walter Reed Army Institute of Research, Silver Spring, MD, USA.

${ }^{11}$ These authors contributed equally: Vincent P. van Hensbergen, Alessandro Ruda. ${ }^{12}$ These authors jointly supervised this work: Nina M. van Sorge and Natalia Korotkova. *e-mail:nsorge3@umcutrecht.nl;nkorotkova@uky.edu 


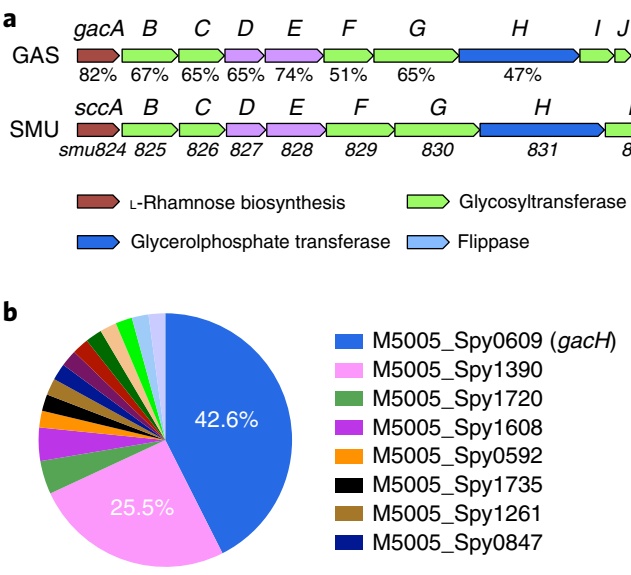

Total 47 hGIIA-resistant mutants
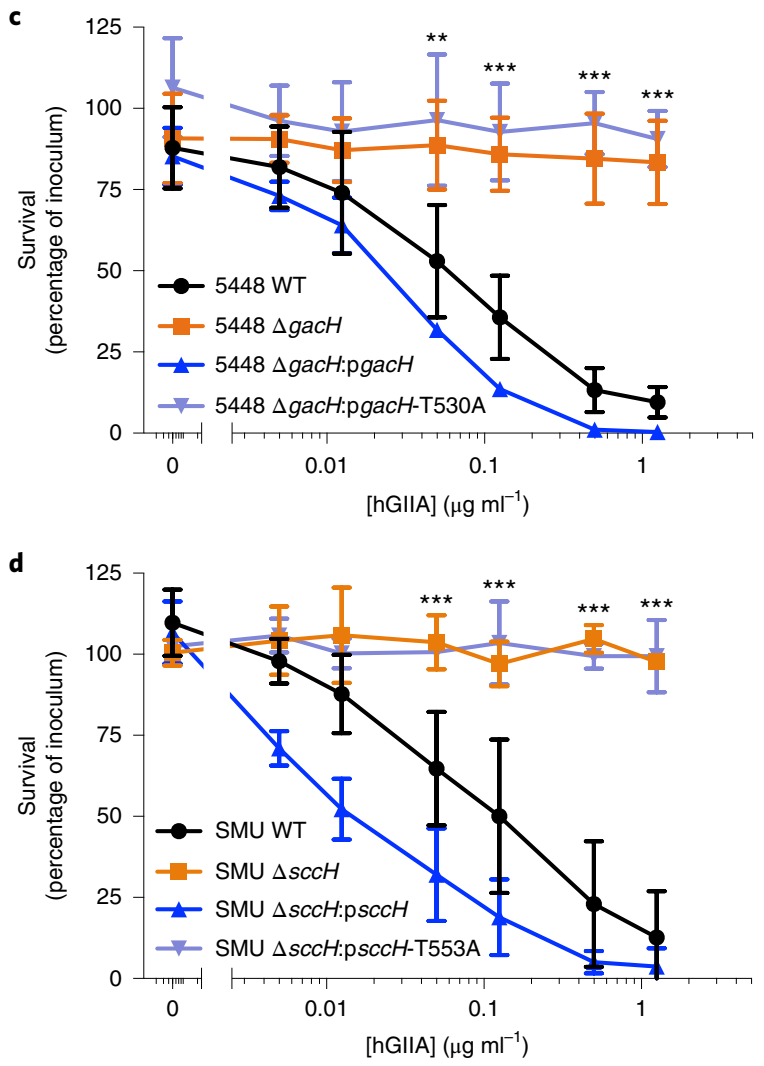

Fig. 1 | GacH homologs are required for hGIIA bactericidal activity against GAS and S. mutans. a, Schematic representation of GAC and SCC biosynthetic gene clusters. SCC biosynthesis encoding gene cluster smu.824-835 was renamed scCABCDEFGHMNPQ. Sequence identity (\%) between encoded homologous proteins is indicated. Sequences of GAS 5005 and S. mutans UA159 were used for identity comparison. b-d, Identification of gacH in transposon sequencing ( $\mathrm{Tn}$-seq) screen and validation for hGIIA resistance. Transposon gene locus tags of the 47 hGIIA-resistant mutants after exposure of Krmit mutant transposon library to lethal concentrations of hGIIA (b). Susceptibility of GAS 5448 and S. mutans to hGIIA concentration range following deletion of $\mathrm{gacH}$ in GAS 5448 (c) and the gacH-homologous gene $\mathrm{sccH}$ (d), respectively. Symbols and error bars represent the mean and s.d., respectively $(n=3$ biologically independent replicates and each replicate represents three technical replicates). $P$ values were calculated by two-way analysis of variance (ANOVA). Bonferroni multiple comparison test was used to statistically compare multiple groups. ${ }^{\star} P<0.05 ;{ }^{\star \star} P<0.01 ;{ }^{\star \star \star} P<0.001$. The precise $P$ values are listed in Supplementary Table 2. identify $g a c H$ as the only valid hit when the Krmit library was exposed to a lethal concentration of hGIIA. Interestingly, $g a c H$ was also identified as a gene providing resistance to zinc toxicity, which is a mechanism deployed by neutrophils to kill $\mathrm{GAS}^{19}$. In pursuit of the underlying mechanism, we have characterized the function of $\mathrm{GacH}$ at the genetic, biochemical and structural level. Our study identifies a previously overlooked GroP modification on both GAC and SCC, and pinpoints GacH homologs as the enzymes responsible for the respective GroP modifications.

\section{Results}

GacH and SccH confer sensitivity to hGIIA. We previously identified $g a c H$ in a GAS Tn-seq transposon library screen as a potential hGIIA susceptibility gene ${ }^{17}$. To identify additional resistant mutants, we exposed the Krmit GAS transposon library ${ }^{16}$ to a lethal concentration of hGIIA. PCR sequencing identified that $43 \%$ of the recovered mutants had a transposon insertion in $\mathrm{gacH}$, and 26\% in $\mathrm{M} 5005$ Spy_1390 (Fig. 1b and Supplementary Table 1). M5005_Spy_1390 was identified in the initial susceptibility screen as an artifact due to biased transposon insertions ${ }^{17}$ and was not investigated further. To validate our finding for $\mathrm{gacH}$, we generated a $\mathrm{gacH}$ deletion mutant in a GAS serotype M1T1 clone 5448, creating 5448 $\mathrm{gacH}$. Deletion of $\mathrm{gacH}$ rendered GAS resistant to hGIIA over the tested concentration range, and was reversed by complementation with $\mathrm{gacH}$ on an expression plasmid (5448 $\mathrm{gacH}$ :pgacH, Fig. 1c). This gacHdependent hGIIA resistance was also observed in two different GAS backgrounds, 2221 (M1T1 clone strain) and 5005 (clinical covS mutant isolate of M1T1 strain) (Supplementary Fig. 1a,b), demonstrating that the effect is conserved across GAS strains of the M1T1 background and independent of CovRS status-a two-component system that regulates about $15 \%$ of the genes in this bacterium ${ }^{20}$.

To investigate whether hGIIA susceptibility was also influenced by $g a c H$ homologs in other streptococci, we deleted $s c c H$ in S. mutans (SMU) serotype $c$ strain Xc, creating SMU $\Delta s c c H$. SMU $\Delta s c c H$ was completely resistant to the tested hGIIA concentrations (Fig. 1d) and susceptibility was restored to wild-type levels by plasmid-expressed $s c \mathrm{cH}$. However, heterologous expression of $g a c H$ in $\mathrm{SMU} \Delta s c c H$ did not restore the phenotype (Supplementary Fig. 1c), suggesting that the enzymes may target different substrates. Altogether, our data indicate that deletion of $\mathrm{gacH}$ homologs renders streptococci more resistant to hGIIA bactericidal activity and that $\mathrm{GacH}$ function is species-specific.

GacH and SccH provide protection from zinc toxicity. Recent evidence indicates that neutrophils deploy zinc poisoning as an antimicrobial strategy against GAS during phagocytosis ${ }^{19}$. To resist $\mathrm{Zn}^{2+}$ toxicity, GAS expresses a zinc efflux system encoded by $c z c D^{19}$. To search for additional $\mathrm{Zn}^{2+}$-resistance genes, we performed a Tn-seq screen of the GAS Krmit transposon library ${ }^{16}$ using two $\mathrm{Zn}^{2+}$ concentrations, 10 and $20 \mu \mathrm{M}$, selected based on growth inhibition analysis (Supplementary Fig. 2a). Genomic DNA for Tn-seq analysis was collected after $T_{2}$ and $T_{3}$ passages (Supplementary Fig. $2 b$ ). In addition to the expected importance of $c z c D$, gacI and $g a c H$ transposon insertions were significantly reduced in the library $(P<0.05)$ after growth with $20 \mu \mathrm{M} \mathrm{Zn}^{2+}$ in both $\mathrm{T}_{2}$ and $\mathrm{T}_{3}$ passages compared to untreated controls, indicating that these genes provide resistance against $\mathrm{Zn}^{2+}$ toxicity (Fig. 2a-d).

To validate our findings, we grew $5448 \Delta \mathrm{gacH}$ and $5448 \Delta \mathrm{gacI}^{11}$ on solid rich medium supplied with different $\mathrm{Zn}^{2+}$ concentrations (Fig. 2e,f). Both mutants showed reduced growth in the medium supplied with $1.25 \mathrm{mM} \mathrm{Zn}^{2+}$, which was restored following complementation with the respective genes (Fig. 2e,f). Again, we checked for function conservation by extending our experiments to S. mutans. Indeed, $\mathrm{SMU} \Delta s c c H$ was more sensitive to $\mathrm{Zn}^{2+}$ in comparison to the parental strain and the phenotype could be restored by $s c c H$ but not by $g a c H$ (Supplementary Fig. 3). Hence, our results 

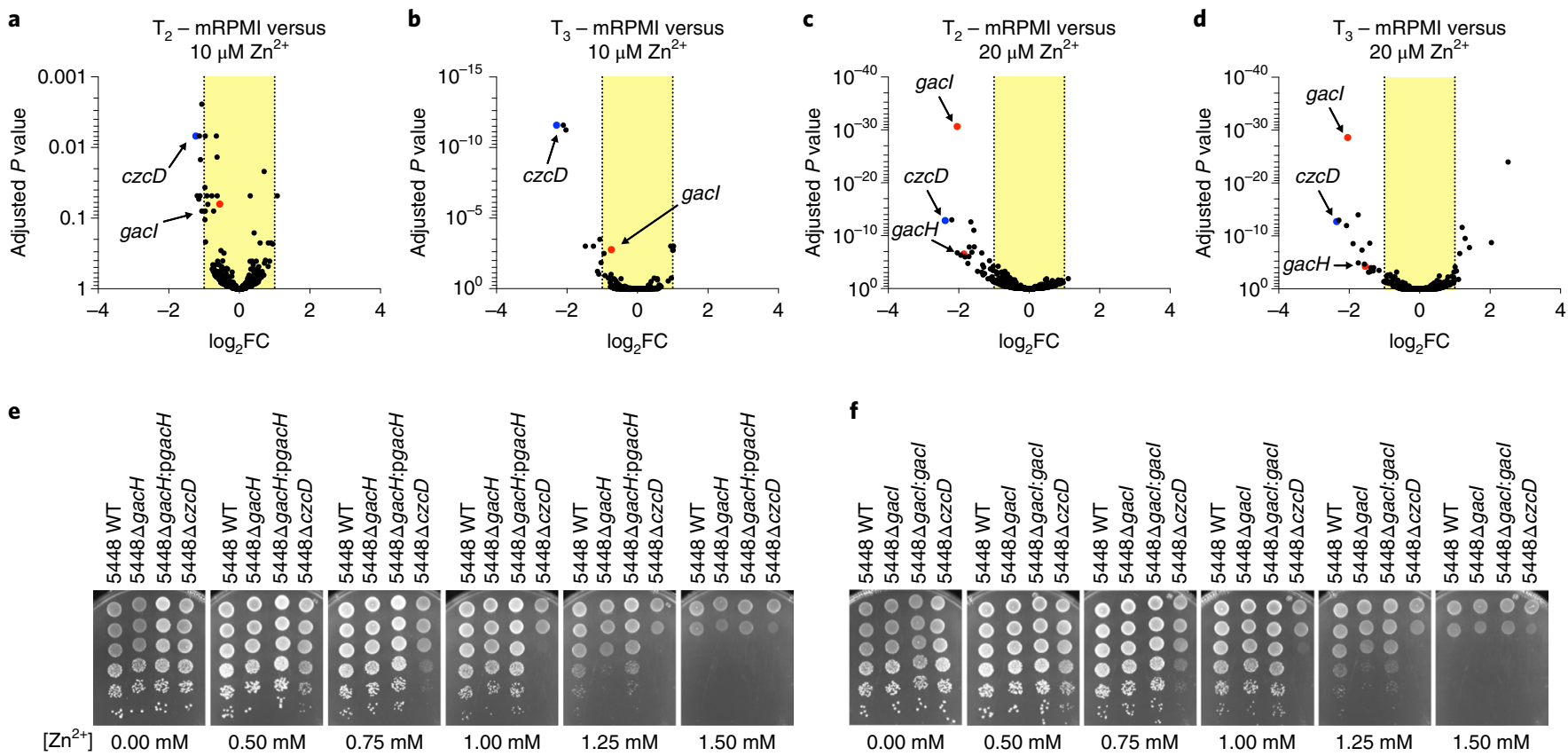

f

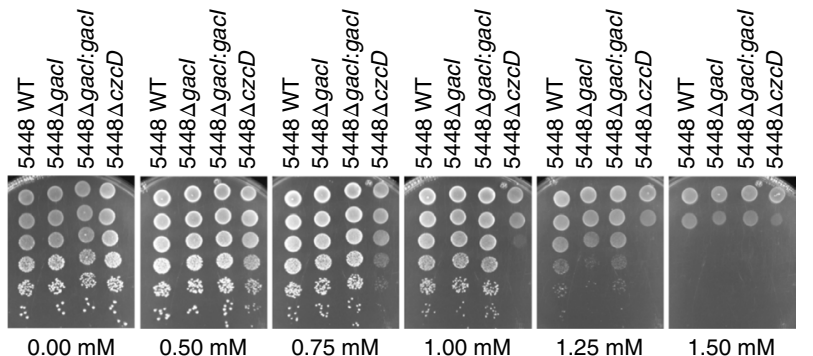

Fig. 2 | Deletion of $\mathbf{g a c l}$ and $\mathbf{g a c H}$ renders GAS susceptible to $\mathbf{Z n}^{\mathbf{2 +}}$. a-d, $\mathrm{Tn}$-seq volcano plots showing representation of $c z c D$, gacH and gacl in GAS Krmit transposon library screens for $Z \mathrm{n}^{2+}$ tolerance. $\log _{2}$ fold change ( $\log _{2} \mathrm{FC}$ ) in fitness was plotted against adjusted $P$ values from Tn-seq analyses through an established pipeline using EdgeR and DEseq2 ( $n=4$ biologically independent replicates used for analysis) ${ }^{47-49}$. The outline of the experiment is shown in Supplementary Fig. 2b. Tn-seq screens of the transposon library were conducted using $10 \mu M \mathrm{Zn}^{2+}$ at $\mathrm{T}_{2}(\mathbf{a}), 10 \mu \mathrm{M} \mathrm{Zn}^{2+}$ at $\mathrm{T}_{3}(\mathbf{b}), 20 \mu \mathrm{M} \mathrm{Zn}^{2+}$ at $\mathrm{T}_{2}(\mathbf{c})$ and

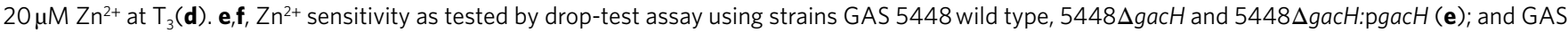
5448 wild type, $5448 \Delta \mathrm{gacl}$ and $5448 \Delta \mathrm{gacl}$ :gacl (f). $5448 \Delta \mathrm{czcD}$ was included as a positive control in both panels. Each drop-test assay experiment was performed independently at least three times and yielded the same results.

provide strong evidence that $\mathrm{GacH}$ and $\mathrm{SccH}$ are important in protecting streptococci from $\mathrm{Zn}^{2+}$ toxicity.

Crystal structure reveals that $\mathrm{GacH}$ is a GroP transferase. $\mathrm{GacH}$ is predicted to contain $11 \mathrm{~N}$-terminal transmembrane segments and an extracellular C-terminal catalytic domain (eGacH). To test the hypothesis that $\mathrm{GacH}$ is a GroP transferase, eGacH was expressed and purified from Escherichia coli. Its crystal structure was determined in apo form (Protein Data Bank (PDB) No. 5U9Z) at $2.0 \AA$ resolution (Fig. 3a,b) and in complex with GroP (PDB No. 6DGM) at $1.49 \AA$ resolution (Fig. 3c). The apo- and GroP-containing eGacH structures belong to different crystal forms, with two molecules in the asymmetric unit. Analysis of the dimer interface and other crystal contacts revealed that the dimer interface has the largest surface of all crystal contacts (1809 and $1894 \AA^{2}$ in the two crystal forms). However, it is scored below the stable complex formation criteria and recombinant $\mathrm{eGacH}$ behaves as a monomer in solution. The structures of the apo- and GroP-bound eGacH monomers are very similar, with root mean square deviation of $0.3 \AA$ for 380 superimposed $\mathrm{C} \alpha$ atoms, as well as between the non-crystallographic copies.

The extracellular C-terminal catalytic domain has an $\alpha / \beta$ core structure that is characteristic for the sulfatase protein family, with the closest similarity to lipoteichoic acid (LTA) synthase $\mathrm{LtaS}^{21,22}$ (Supplementary Figs. 4a,b and 5) and LTA primase $\mathrm{LtaP}^{23}$ (Supplementary Table 3). LtaS and LtaP are GroP transferases that participate in biosynthesis of LTA, a crucial constituent of Gram-positive cell envelopes, consisting of a poly(GroP) backbone linked to a glycolipid membrane anchor ${ }^{24}$. The catalytic site of eGacH contains a $\mathrm{Mn}^{2+}$ ion coordinated by residues E488, T530, D711 and H712, equivalent to residues E255, T300, D475 and H476 of a C-terminal extracellular domain of LtaS (eLtaS) from Staphylococcus aureus (Fig. 3c and Supplementary Figs. 4c,d and 5). The structure of eGacH in complex with GroP revealed the position of the ligand in the active site with the phosphoryl group oriented towards the $\mathrm{Mn}^{2+}$ ion, and coordinated by residues G529, T530 and H650 (Fig. 3c). The glycerol 2- and 3-hydroxyl groups form hydrogen bonds with side-chains of residues R589, H580 and N586. The positions of GroP and coordinating residues are similar in $\mathrm{eGacH}$ and $S$. aureus eLtaS structures. For example, the glycerol moiety forms hydrogen bonds with residues $\mathrm{H} 580$ and $\mathrm{R} 589$ in $\mathrm{GacH}$ and equivalent residues $\mathrm{H} 347$ and R356 in S. aureus eLtaS (Fig. 3c and Supplementary Fig. $4 c, d)^{21}$. Thus, the structure of eGacH in complex with GroP is consistent with the idea that GacH and LtaS use related catalytic mechanisms to transfer GroP to substrates.

To functionally assess the requirement of the catalytic residues, we examined the bactericidal activity of hGIIA in $5448 \Delta \mathrm{gacH}$ and $\mathrm{SMU} \Delta s c \mathrm{H}$ expressing catalytically inactive versions of $\mathrm{gacH}$ and $s c c H$, in which the active site T530 and T533 codons were replaced by alanine, respectively (Supplementary Fig. 6). The non-functional $g a c H$ and $s c c H$ did not restore hGIIA susceptibility (Fig. 1c,d), indicating that the GroP transferase activity of the $\mathrm{gacH}$ and $s c \mathrm{H}$ gene products is required for the observed hGIIA-dependent phenotypes.

GacH cleaves phosphatidylglycerol to release GroP. Experimental evidence suggests that LtaS utilizes the GroP head group of the membrane lipid phosphatidylglycerol as donor for poly(GroP) backbone biosynthesis, liberating diacylglycerol ${ }^{24,25}$. To assess whether $\mathrm{GacH}$ also catalyzes the cleavage of phosphatidylglycerol to yield GroP for a transfer reaction, we performed an in vitro experiment employing the $\mathrm{eGacH}$ protein and a fluorescently labeled artificial substrate, NBD-phosphatidylglycerol. Incubation of eGacH with NBD-phosphatidylglycerol yielded a fluorescent product (Supplementary Fig. 7a) with the same mobility on silica gel thin-layer chromatography as NBD-diacylglycerol, which was obtained from NBD-phosphatidylglycerol by enzymatic cleavage with phospholipase $\mathrm{C}$ from Bacillus cereus. Furthermore, the 
a

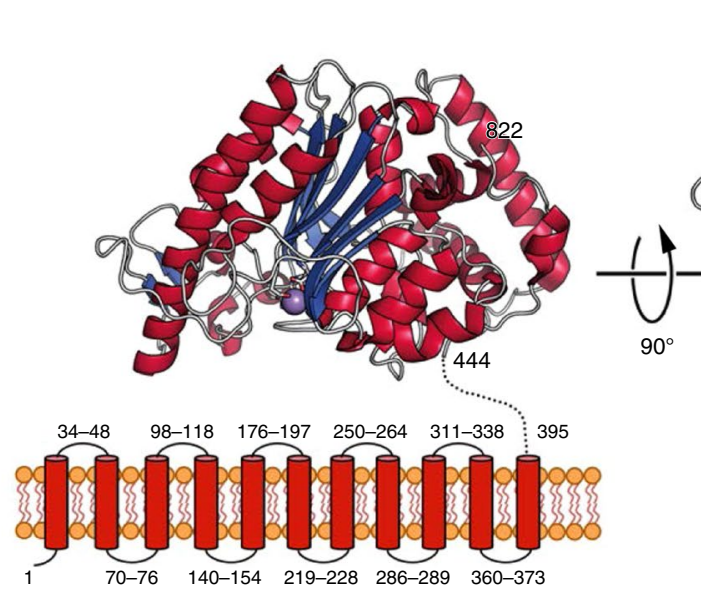

b

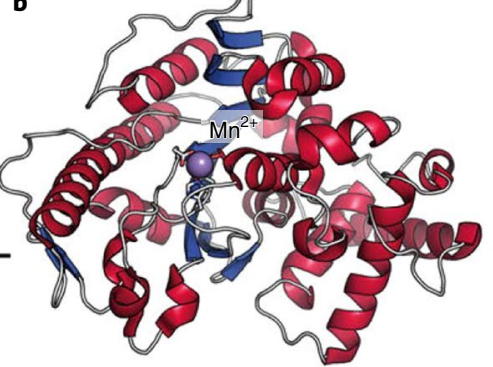

GAS GacH

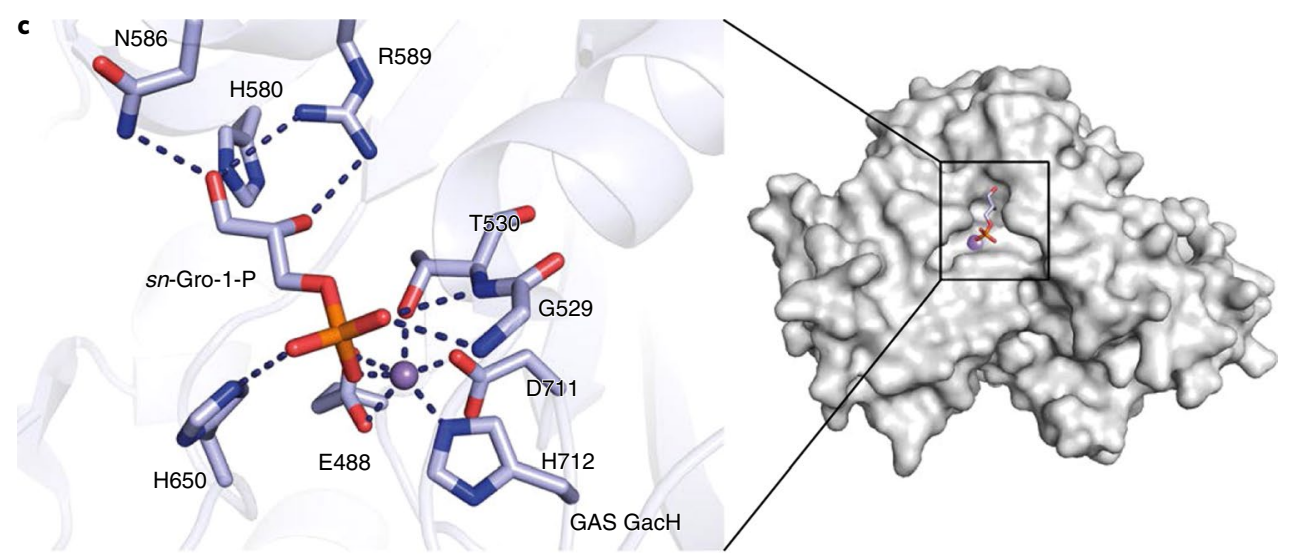

Fig. 3 | Structure of eGacH. a, Predicted topology of GacH showing 11 transmembrane helices and structure of extracellular domain with the enzymatic active site oriented toward the cell membrane. $\mathbf{b}$, Structure of apo eGacH viewed at the active site with the $\mathrm{Mn}^{2+}$ ion shown as a violet sphere.

c, A close-up view of the active site $\mathrm{GacH}$ crystal structure in complex with sn-Gro-1-P.

eGacH product recovered from the silica gel plate and analyzed by liquid chromatography-mass spectrometry (LC-MS) yielded a spectrum consistent with NBD-diacylglycerol and identical to that of the phospholipase $\mathrm{C}$ product (Supplementary Fig. $7 \mathrm{~b}-\mathrm{d}$ ). The formation of NBD-diacylglycerol by eGacH required the active-site residues, since the eGacH variant, eGacH-T530A, was not active in the assay (Supplementary Fig. 7a). These observations are consistent with the assignment of $\mathrm{GacH}$ as a GroP transferase.

GacH homologs decorate glycopolymers with GroP. Phylogenetic analysis of either the full-length or extracellular domains of $\mathrm{GacH}$ homologs and LtaS-related proteins revealed that these proteins fall into distinct clades of GroP transferases, suggesting that the proteins may transfer GroP to different substrates (Supplementary Fig. 8). To assess whether $\mathrm{gacH}$ homologs modify the respective streptococcal glycopolymers with GroP, we enzymatically released GAC and SCC from purified cell walls from GAS and S. mutans strains. Subsequently, the enriched polysaccharide preparations were analyzed for glycerol and phosphate. Hydrolysis with $\mathrm{HCl}$ released a large amount of glycerol from GAC and SCC isolated from wildtype bacteria (Fig. 4a,b and Supplementary Fig. 9a). Furthermore, we detected high levels of inorganic phosphate after incubation of these acid-treated samples with alkaline phosphatase (Fig. $4 \mathrm{a}$,b and Supplementary Fig. 9a), which was not detected when intact GAC was treated with alkaline phosphatase (Supplementary Fig. 9b,c). This indicates that the phosphoryl moiety is present as a phosphodiester, consistent with its identification as GroP. In contrast to wildtype GAC and SCC, the glycopolymers isolated from $5448 \Delta \mathrm{gacH}$,
$5005 \Delta g a c H$ and SMU $\Delta s c c H$ contained a markedly reduced amount of glycerol and phosphate (Fig. 4a,b and Supplementary Fig. 9a), which was restored only by complementation with wild-type $\mathrm{gacH}$, but not $g a c H$-T530A, for GAS (Fig. 4a) or plasmid-expressed $s c c H$ for SMU (Fig. 4b).

In accordance with our functional data, expression of $\mathrm{gacH}$ did not restore the glycerol and phosphate levels in SCC of SMU $\Delta s c c H$ (Fig. 4b). This suggested that GroP modifications might involve the species-specific side-chains (Glc versus GlcNAc) rather than the identical polyrhamnose backbone. Indeed, the glycerol and phosphate contents in GAC isolated from the GlcNAc-deficient mutant, $5448 \Delta$ gacI, were significantly reduced (Fig. 4a). Importantly, analysis of GAS strains for total carbohydrate, phospholipid and phosphatidylglycerol contents established that deletion of $\mathrm{gacH}$ had no effect on these components (Supplementary Fig. 10a-c). Furthermore, the semi-quantitative immuno-dot-blot analysis of GAS strains with anti-GAC antibodies demonstrated that the absolute amount of GAC is not affected by gacH deletion (Supplementary Fig. 10d). Analysis of the glycosyl composition of purified cell walls demonstrated that the absence of $\mathrm{GacH}$ and $\mathrm{SccH}$ did not affect the Rha/ GlcNAc and Rha/Glc ratios, respectively (Supplementary Fig. 10e,f).

To provide further evidence that GAC is modified with GroP, GAC samples were subjected to alkaline hydrolysis to release GroP, as described in ref. ${ }^{26}$, and the hydrolysate was analyzed by LC-MS for high-molecular weight fragment ions arising from GroP. Compared to wild type, deletion of either $g a c H$ or $g a c I$ markedly reduced the levels of GroP in GAC (Supplementary Fig. 11). Complementation of $5448 \mathrm{gacH}$ with native $\mathrm{gacH}$, but not with inactive $\mathrm{gacH}$-T530A, 


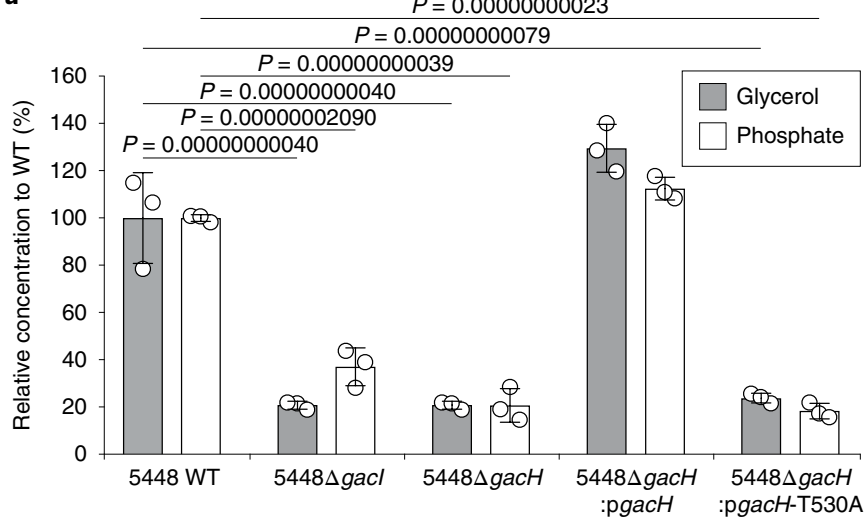

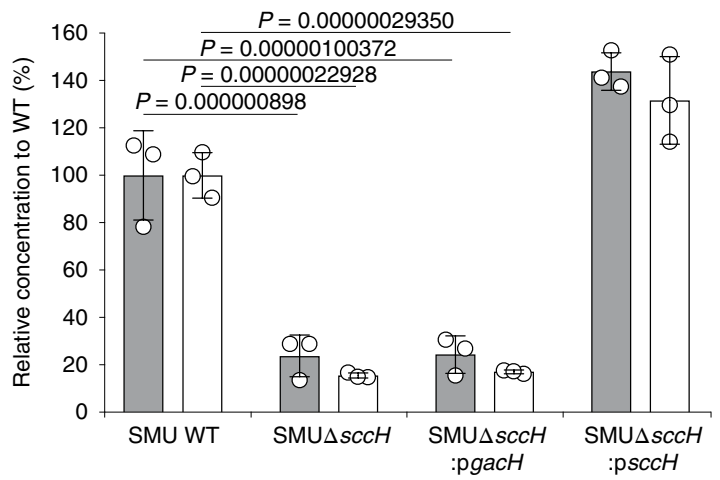
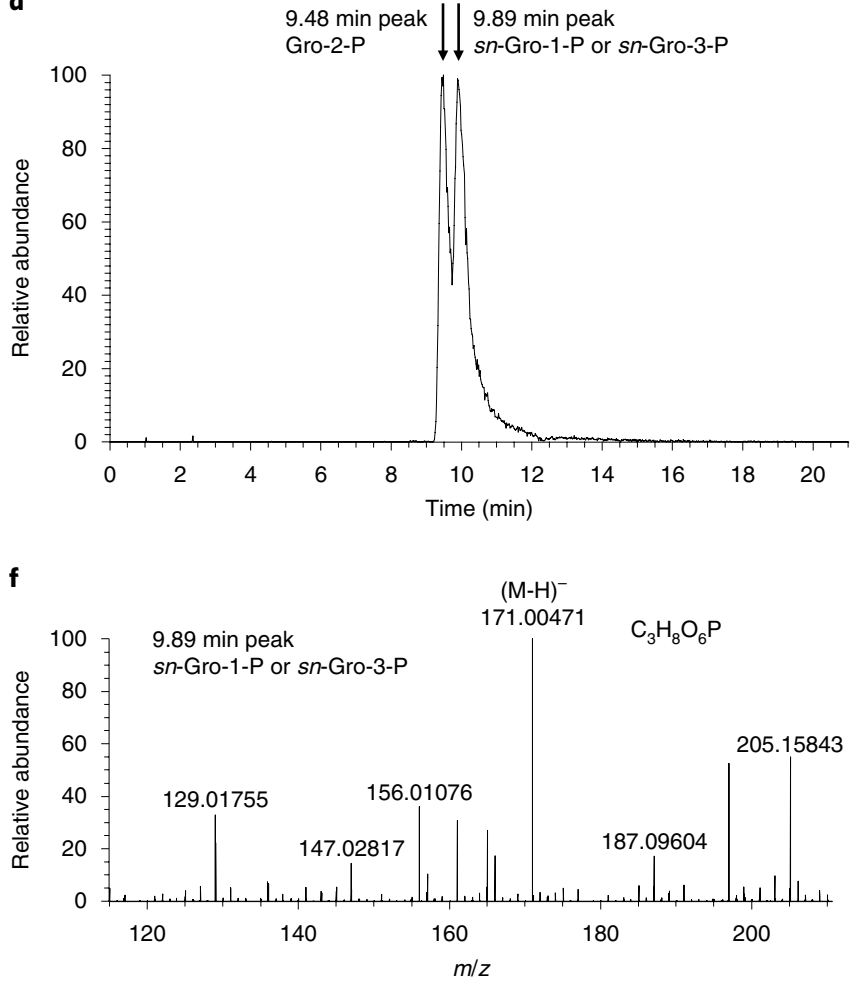

d
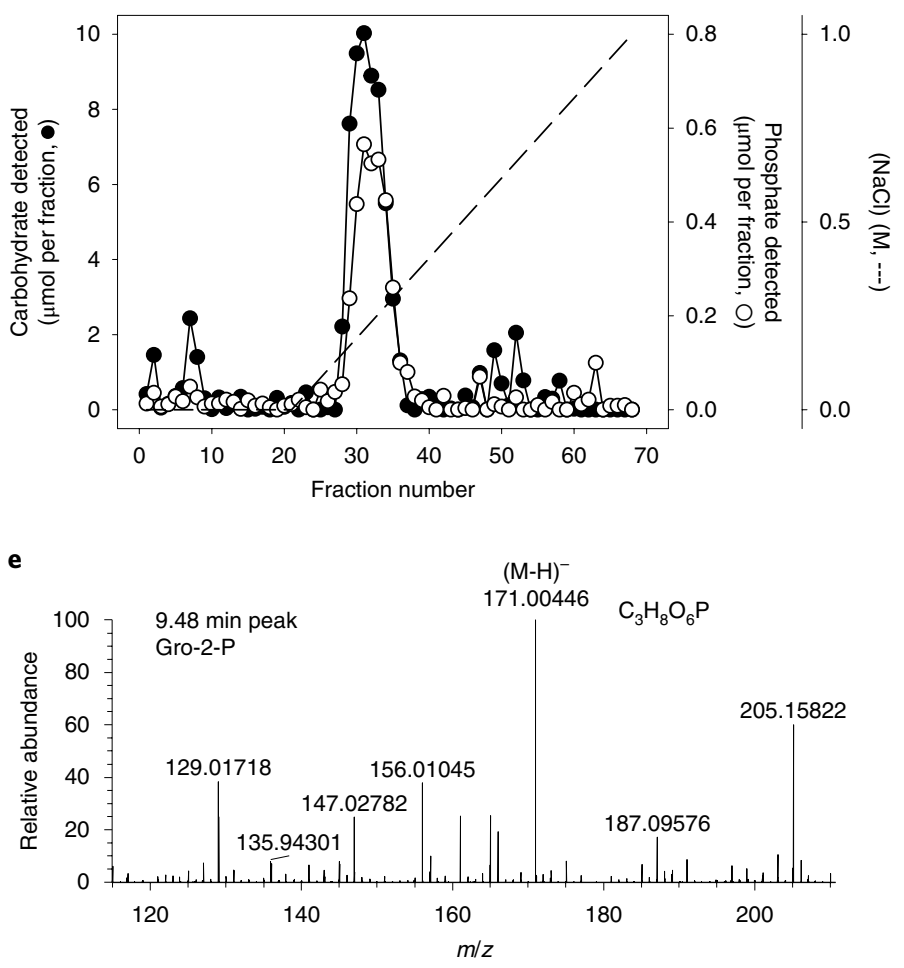

Fig. 4 | GacH and SccH modify their respective glycopolymers with sn-Gro-1-P. a,b, Analysis of glycerol and phosphate content in GAC and SCC isolated from GAS 5448 wild type, $\Delta$ gacl, $\Delta$ gacH and $\Delta$ gacH complemented with native gacH or a catalytically inactivated version of gacH (gacH-T530A) (a), and S. mutans wild type, $\Delta \mathrm{sccH}$ and $\Delta \mathrm{sccH}$ complemented with sccH or gacH (b). The concentrations of phosphate and glycerol are presented relative to the wild-type strain. Bars and error bars represent the average and s.d., respectively ( $n=3$ biologically independent samples). $P$ values were calculated and adjusted by two-way ANOVA and Bonferroni's multiple comparison test. c, Diethylaminoethanol (DEAE)-Sephacel elution profile of GAC isolated from $\sim 90 \mathrm{mg}$ of GAS cell wall. Fractions were analyzed for carbohydrate $(\bullet)$ and phosphate $(\mathrm{O})$. $\mathbf{d}-\mathbf{f}$, Identification of the enantiomeric form of GroP associated with GAC. $\mathbf{d}$, The GroP isomers were recovered from GAC following alkaline hydrolysis and separated by liquid chromatography as outlined in Methods. The elution positions corresponding to standard Gro-2-P and sn-Gro-1-P/sn-Gro-3-P are indicated by the arrows. LC-MS analysis identified two extracted ion chromatogram peaks for the molecular GroP ion mass/charge number $(\mathrm{m} / \mathrm{z}) 171.004[\mathrm{M}-\mathrm{H}]^{-}$, which eluted at $9.48(\mathbf{e})$ and 9.89 min $(\mathbf{f})$. Based on the accurate mass and retention times, these two peaks were assigned as Gro-2-P and sn-Gro-1-P/sn-Gro-3-P, respectively, by comparison with authentic chemical standards. Experiments depicted in c-f were performed independently twice and yielded the same results.

fully restored GroP levels (Supplementary Fig. 11). Thus, the differences in GroP content for the $\mathrm{gacH}$ and $\mathrm{gacI}$ deletion mutants are consistent with a role for $\mathrm{GacH}$ in modification of the GlcNAc side-chain of GAC with GroP.

To show that GroP is attached directly to GAC, the wild-type GAC was further purified by a combination of size-exclusion and ion-exchange chromatography (Fig. 4c and Supplementary Fig. 12a). The majority of the rhamnose- and phosphate-containing material was bound to the ion-exchange column and eluted as a single coincident peak (Fig. 4c). The GAC purified from 5005 $\mathrm{gacH}$ did not bind to the column (Supplementary Fig. 12b). Interestingly, the 5005 $\Delta$ gacH GAC does appear to contain a small amount of phosphate that may have arisen from the phosphodiester bond linking GAC to peptidoglycan. Taken together, our data directly support the conclusion that GAC is modified with GroP donated by $\mathrm{GacH}$. 
GacH decorates GAC with sn-Gro-1-P. To assess which GroP enantiomer was incorporated in GAC, GroP liberated from purified GAC by alkaline hydrolysis was analyzed further (Supplementary Fig. 13). As described in detail in ref. ${ }^{26}$, if GAC is modified by snGro-1-P, alkaline hydrolysis of the phosphodiester bond should result in the formation of a mixture of $s n$-Gro-1-P and Gro2-P, whereas modification by $s n$-Gro-3-P would yield a mixture of $s n$-Gro-3-P and Gro-2- $\mathrm{P}^{26}$. As expected, LC-MS analysis of GAC-derived GroP revealed the presence of two GroP isomers of approximately equal proportions, with LC retention times and major high-molecular weight ions consistent with standard $s n$-Gro1-P/sn-Gro-3-P and Gro-2-P (Fig. 4d-f and Supplementary Fig. 14). The recovered GroP was characterized further by enzymatic analysis using an $s n$-Gro-3-P assay kit. Under reaction conditions in which $s n$-Gro-3-P standard produced a robust enzymatic signal, incubation with an equal amount of either $s n-G r o-1-P$ or the unknown GroP resulted in negligible activity (Supplementary Fig. 15). When $s n$-Gro-3-P was mixed with an equal amount of either $s n-G r o-1-\mathrm{P}$ or the unknown mixture of GroP isomers, 85.8 and $90.0 \%$ of the activity detected with the standard $s n$-Gro-3-P alone was found, confirming that the negative result using the unknown mixture was not due to the presence of an inhibitory compound in GroP preparation. Taken together, our results indicate that $\mathrm{GacH}$ decorates GAC with $s n$-Gro-1-P, which is most probably derived from phosphatidylglycerol.

GroP is attached to the C6 hydroxyl group of GlcNAc. To unambiguously establish the presence and location of GroP in GAC, the glycopolymer was isolated from wild-type GAS and analyzed by nuclear magnetic resonance (NMR) (Fig. 5a, Supplementary Table 4 and Supplementary Figs. 16 and 17). The details of NMR analysis are described in Supplementary Notes. Wild type GAC is partially substituted by a GroP residue at $\mathrm{O} 6$ of the side-chain $\beta$-D-GlcpNAc residue; based on integration of the cross-peaks for the anomeric resonances in the ${ }^{1} \mathrm{H},{ }^{13} \mathrm{C}$-HSQC NMR spectrum, the GAC preparation carries GroP groups to $\sim 25 \%$ of the GlcNAc residues. To validate the two-dimensional (2D) NMR results, a triple-resonance ${ }^{1} \mathrm{H},{ }^{13} \mathrm{C},{ }^{31} \mathrm{P}$ NMR experiment based on through-bond ${ }^{1} J_{\mathrm{HC}}$ as well as ${ }^{2} J_{\mathrm{CP}}$ and ${ }^{3} J_{\mathrm{CP}}$ correlations ${ }^{27}$ was carried out. The three-dimensional (3D) NMR experiments revealed the ${ }^{1} \mathrm{H}$ NMR chemical shifts of $\mathrm{H}^{\prime}$ ' and the two $\mathrm{H}^{\prime}$ protons of the $\beta$-D-GlcpNAc residue, as well as the two $\mathrm{H} 1$ protons and $\mathrm{H} 2$ of the Gro residue that all correlated to ${ }^{13} \mathrm{C}$ nuclei (Fig. 5b). The ${ }^{13} \mathrm{C}$ NMR chemical shifts of $\mathrm{C} 5$ and $\mathrm{C}^{\prime}$ of the $\beta$-D-GlcpNAc residue, as well as C1 and C2 of the Gro residue, all correlated to the ${ }^{31} \mathrm{P}$ nucleus (Fig. 5b), and the above protons correlated to the ${ }^{31} \mathrm{P}$ nucleus (Fig. 5b). Taking into consideration the $\mathrm{GacH}$-mediated mechanism of GAC modification by GroP, as well as the biochemical experiments carried out herein, the substituent at $\mathrm{O} 6$ of $\beta-\mathrm{D}-\mathrm{Glc} p \mathrm{NAc}$ is an $s n$-Gro-1-P group (Fig. 5c).

\section{Discussion}

In Gram-positive bacteria, many peptidoglycan-attached glycopolymers contain negatively charged groups in the repeating units ${ }^{2}$. Previous detailed studies deduced the chemical structure of glycopolymers from GAS and S. mutans ${ }^{3-5}$. However, none identified anionic groups in these structures except for one study that reported the presence of glycerol and phosphate in GAC and proposed that this GroP is part of the phosphodiester linkage connecting GAC to peptidoglycan ${ }^{28}$. Similarly, other reports identified substantial concentrations of phosphate in the glycopolymers isolated from a number of streptococcal species ${ }^{29-31}$. Phosphate detection was either disregarded as contamination with LTA $^{29}$ or further analyzed using ${ }^{1} \mathrm{HNMR}$ or ${ }^{13} \mathrm{CNMR}$ methods ${ }^{5,31,32}$ that do not directly detect phosphoryl moieties in polysaccharides. In our report, we unambiguously confirm that the glycopolymers of GAS and S. mutans are in fact polyanionic molecules through decoration of their respective glycan side-chains with GroP (Fig. 5c,d).

We identified and structurally characterized an alternative class of GroP transferase enzymes, represented by $\mathrm{GacH}$, which modifies GAC with GroP in the human pathogen GAS. According to our phylogenetic analysis, $\mathrm{GaCH}$ homologs are present in many streptococci (Supplementary Fig. 8), suggesting that these bacteria express glycopolymers with GroP-modified side-chains, as we have demonstrated here for S. mutans. GacH belongs to the alkaline phosphatase superfamily of which two GroP transferases involved in LTA synthesis, LtaS and LtaP, have been biochemically and structurally characterized ${ }^{21-23,33}$. LtaS and LtaP are membrane proteins that use the membrane lipid phosphatidylglycerol as the GroP donor for the transfer reaction ${ }^{25}$. Our structural analysis of $\mathrm{GacH}$ in complex with GroP indicates that the T530 residue participates in the formation of a GroP-enzyme intermediate similar to observations in LtaS, where the GroP molecule is complexed in the active site threonine residue which functions as a nucleophile in phosphatidylglycerol hydrolysis ${ }^{21-23}$. The importance of this residue was also confirmed functionally, since complementation of $\mathrm{gacH}$ mutant strains with a T530A $\mathrm{gacH}$ variant could not restore GroP content in GAC and hGIIA sensitivity to wild-type levels. The observations, that the extracellular domain of $\mathrm{GacH}$ cleaves phosphatidylglycerol and the GroP in GAC is the sn-Gro-1-P enantiomer, strongly suggest that $\mathrm{GacH}$ uses phosphatidylglycerol as its donor substrate for the transfer reaction, similar to LtaS (Fig. 5d).

In Gram-positive bacteria, the modification of teichoic acids with $\mathrm{D}$-alanine provides resistance against antibiotics, cationic antimicrobial peptides and small bactericidal enzymes including hGIIA, and affects $\mathrm{Mg}^{2+}$ ion scavenging ${ }^{1,2,34}$. It has been assumed that incorporation of positively charged $\mathrm{D}$-alanine into teichoic acids decreases negative bacterial surface charge resulting in reduced initial binding of cationic antimicrobial peptides to the bacterial surface due to ionic repulsion ${ }^{35,36}$. Our study demonstrates that addition of the negatively charged GroP group to glycopolymers protects streptococci from zinc toxicity but also renders bacteria more sensitive to hGIIA activity.

A large body of evidence indicates that phagocytic cells utilize $\mathrm{Zn}^{2+}$ intoxication to suppress the intracellular survival of bacteria ${ }^{37}$. Zinc is essential as a key catalytic or structural element for a wide variety of proteins and its concentration needs to be maintained at a specific level, which requires sophisticated systems for uptake and efflux of metal ions. Hence elevated levels of zinc in the cytosol result in cellular toxicity ${ }^{19}$, which for GAS is due to inhibition of central carbon metabolism ${ }^{38}$. One mechanism of microbial susceptibility to zinc toxicity is mediated by extracellular competition of $\mathrm{Zn}^{2+}$ for $\mathrm{Mn}^{2+}$ transport and thereby mediates toxicity by impairing acquisition of $\mathrm{Mn}^{2+}$, the essential nutrient metal ${ }^{39}$. Accordingly, the phenotypes of our GroP and GlcNAc side-chain-deficient mutants could be explained either by 'trapping' of $\mathrm{Zn}^{2+}$ in the wild-type cell wall by GroP, or the increased $\mathrm{Mn}^{2+}$-binding capacity of GroP-modified bacterial cell wall. which has been proposed to act as the conduit for the trafficking of mono- and divalent cations to the membrane ${ }^{34}$.

Charge-dependent mechanisms probably underlie the increased hGIIA susceptibility of GAS and S. mutans expressing GroPmodified glycopolymers. hGIIA is a highly cationic enzyme that catalyzes the hydrolysis of bacterial phosphatidylglycerol ${ }^{40,41}$, ultimately leading to bacterial death through lysis. Traversal of this bactericidal enzyme through the Gram-positive cell wall to the plasma membrane is charge-dependent. Indeed, the absence of D-alanine modifications in teichoic acids severely compromises S.aureus survival when challenged with hGIIA ${ }^{41,42}$. Similarly, the GacH/SccHdependent GroP modifications on glycopolymers are required for hGIIA to exert its bactericidal effect against GAS and S. mutans, respectively. We have previously demonstrated that loss of the entire GlcNAc GAC side-chain strongly hampers hGIIA trafficking 


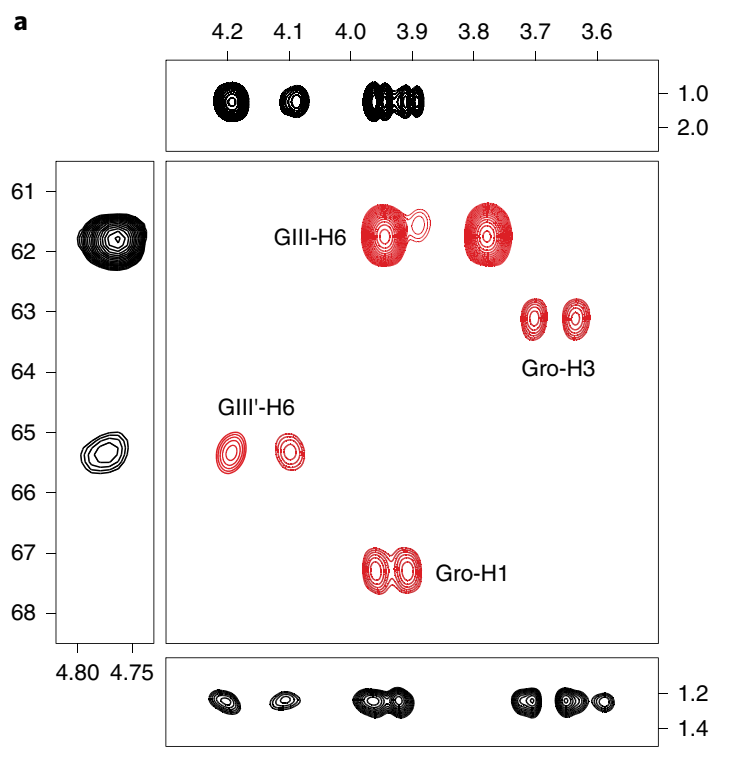

b

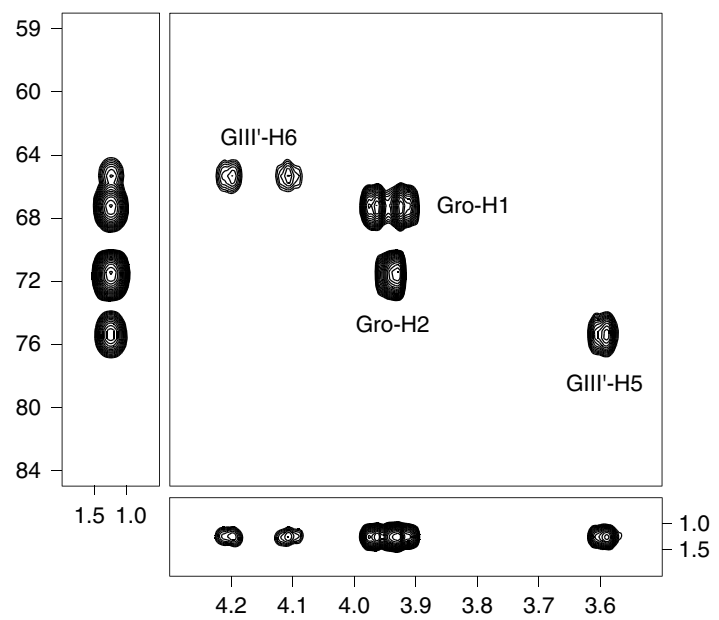

c

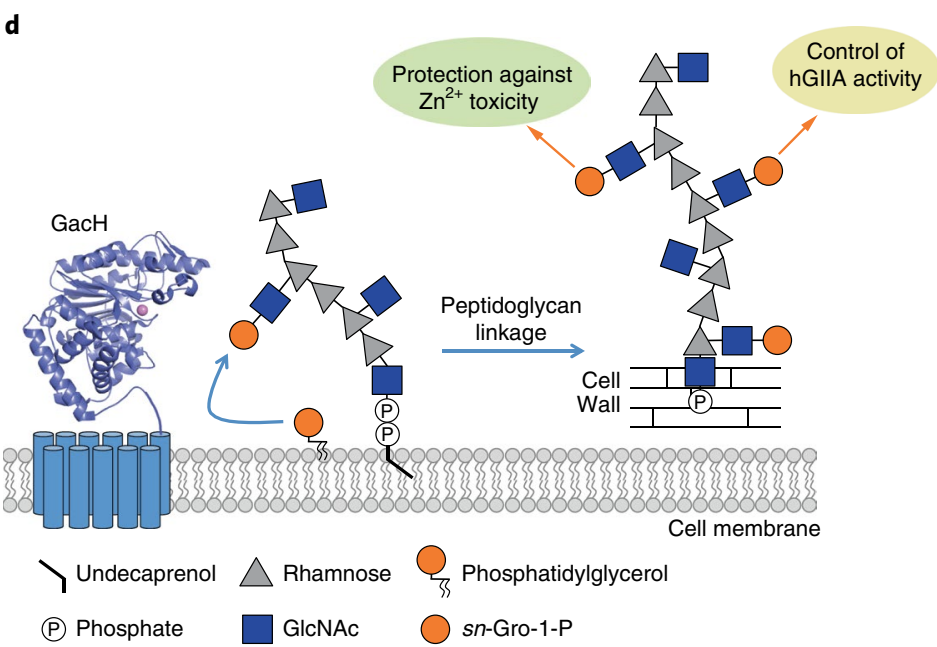

Fig. 5 | NMR analysis confirms the presence of GroP on the C6 GlcNAc hydroxymethyl group of GAC. $\mathbf{a}, \mathbf{b}$, Selected regions of NMR spectra of GAC. a, Multiplicity-edited ${ }^{1} \mathrm{H}_{,}^{13} \mathrm{C}-\mathrm{HSQC}$ in which methylene groups have opposite phase and are shown in red (center box); ${ }^{1} \mathrm{H}^{13}{ }^{13} \mathrm{C}-\mathrm{HSQC}$-TOCSY with an isotropic mixing time of $120 \mathrm{~ms}$ (left box); ${ }^{1} \mathrm{H}_{1}{ }^{13} \mathrm{C}-\mathrm{HMBC}$ with a mixing time of $90 \mathrm{~ms}$ (top box); ${ }^{1} \mathrm{H},{ }^{31} \mathrm{P}$-hetero-TOCSY with an isotropic mixing time of $80 \mathrm{~ms}$ (bottom box). b, ${ }^{1} \mathrm{H},{ }_{1}^{13} \mathrm{C}$-plane (center box); ${ }^{13} \mathrm{C},{ }^{31} \mathrm{P}$-plane using a nominal ${ }^{n} \mathrm{JP}_{\mathrm{CP}}$ value of $5 \mathrm{~Hz}$ (left box); ${ }^{1 \mathrm{H}},{ }^{31 \mathrm{P}}$-plane (bottom box) of a through-bond $3 \mathrm{D}{ }^{1} \mathrm{H}_{1}^{13} \mathrm{C}_{1}^{13} \mathrm{P}$ NMR experiment. Cross-peaks are annotated as GIII corresponding to the GIcNAc residue, GIII' being the GroP-substituted GlcNAc residue and Gro the glycerol residue. NMR chemical shifts of ${ }^{1} \mathrm{H}$ (horizontal axis), ${ }^{13} \mathrm{C}$ (left axis) and ${ }^{31} \mathrm{P}$ (right axis and left box in $\mathbf{b}$ ) are given in ppm. Experiments depicted in $\mathbf{a}, \mathbf{b}$ were performed independently three times and yielded the same results. $\mathbf{c}$, Schematic structure of the GAC repeating unit consisting of $\rightarrow 3$ )- $\alpha-L-R h a p-(1 \rightarrow 2)[\beta-D-G l c p N A c 6 P(S)$ Gro- $(1 \rightarrow 3)]-\alpha-L-R h a p-(1 \rightarrow . \mathbf{d}$, The mechanism and roles of GroP cell wall modification in streptococci.

through the GAS cell wall, with a minor contribution of reduced hGIIA binding to the cell surface ${ }^{17}$. Since GroP modifications were also lost in the GlcNAc side-chain-deficient mutant, 5448 $\Delta$ gacI, described in this study, we now assume that the mechanisms of the hGIIA-dependent phenotype are similar in the gacI and gacH mutants.

Another very important aspect of our study is the identification of an alternative, potentially antigenic, epitope on the surface of streptococci. GAS is associated with numerous mild to life-threatening invasive diseases ${ }^{43}$ and is also causative of post-infectious sequelae, including rheumatic heart disease ${ }^{43}$. In particular, the invasive manifestations and post-infectious sequelae are difficult to treat with antibiotics and a GAS vaccine is urgently needed to combat these neglected diseases. The GAC is an attractive candidate for GAS vaccine development, due to its conserved expression in all GAS serotypes and the absence of the constitutive component of GAC, Rha, in humans ${ }^{12,13}$. However, it has been proposed that the GAC GlcNAc side-chain may elicit cross-reactive antibodies relevant to the pathogenesis of rheumatic fever and rheumatic heart disease $^{44-46}$. Moreover, persistence of anti-GAC and anti-GlcNAc antibodies is associated with a poor prognosis in rheumatic heart disease ${ }^{45}$. These clinical associations and the lack of understanding of the pathogenesis of GAS post-infectious rheumatic heart disease have hampered progress in the development of GAC-based vaccines against GAS. However, the GAC GlcNAc decorated with GroP might be an attractive candidate for GAS vaccine development because GroP-modified GlcNAc represents a unique epitope that is absent from human tissues. Thus, our study has implications for the design of a safe and effective vaccine against this important human pathogen for which a vaccine is currently lacking. 


\section{Online content}

Any methods, additional references, Nature Research reporting summaries, source data, statements of data availability and associated accession codes are available at https://doi.org/10.1038/ s41589-019-0251-4.

Received: 8 July 2018; Accepted: 20 February 2019;

Published online: 1 April 2019

\section{References}

1. Brown, S., Santa Maria, J. P. Jr. \& Walker, S. Wall teichoic acids of Grampositive bacteria. Annu. Rev. Microbiol. 67, 313-336 (2013).

2. Weidenmaier, C. \& Peschel, A. Teichoic acids and related cell-wall glycopolymers in Gram-positive physiology and host interactions. Nat. Rev. Microbiol. 6, 276-287 (2008).

3. Mistou, M. Y., Sutcliffe, I. C. \& van Sorge, N. M. Bacterial glycobiology: rhamnose-containing cell wall polysaccharides in Gram-positive bacteria. FEMS Microbiol. Rev. 40, 464-479 (2016).

4. Huang, D. H., Rama Krishna, N. \& Pritchard, D. G. Characterization of the group A streptococcal polysaccharide by two-dimensional ${ }^{1} \mathrm{H}$-nuclearmagnetic-resonance spectroscopy. Carbohydr. Res. 155, 193-199 (1986).

5. St Michael, F. et al. Investigating the candidacy of the serotype specific rhamnan polysaccharide based glycoconjugates to prevent disease caused by the dental pathogen Streptococcus mutans. Glycoconj. J. 35, 53-64 (2018).

6. Van der Beek, S. L. et al. A Streptococcus and defines a new class of monomeric dTDP-4-dehydrorhamnose reductases (RmlD). Mol. Microbiol. 98, 946-962 (2015).

7. Tsuda, H., Yamashita, Y., Shibata, Y., Nakano, Y. \& Koga, T. Genes involved in bacitracin resistance in Streptococcus mutans. Antimicrob. Agents Chemother 46, 3756-3764 (2002).

8. De, A. et al. Deficiency of RgpG causes major defects in cell division and biofilm formation, and deficiency of LytR-CpsA-Psr family proteins leads to accumulation of cell wall antigens in culture medium by Streptococcus mutans. Appl. Environ. Microbiol. 83, e00928 (2017).

9. Nagata, E. et al. Serotype-specific polysaccharide of Streptococcus mutans contributes to infectivity in endocarditis. Oral Microbiol. Immunol. 21, $420-423$ (2006).

10. Henningham, A. et al. Virulence role of the GlcNAc side chain of the Lancefield cell wall carbohydrate antigen in non-M1-serotype group A Streptococcus. mBio 9, e02294 (2018).

11. van Sorge, N. M. et al. The classical Lancefield antigen of group A Streptococcus is a virulence determinant with implications for vaccine design. Cell Host Microbe 15, 729-740 (2014).

12. Kabanova, A. et al. Evaluation of a group A Streptococcus synthetic oligosaccharide as vaccine candidate. Vaccine 29, 104-114 (2010).

13. Sabharwal, H. et al. group A Streptococcus (GAS) carbohydrate as an immunogen for protection against GAS infection. J. Infect. Dis. 193, 129-135 (2006).

14. Shibata, Y., Yamashita, Y., Ozaki, K., Nakano, Y. \& Koga, T. Expression and characterization of streptococcal rgp genes required for rhamnan synthesis in Escherichia coli. Infect. Immun. 70, 2891-2898 (2002).

15. Rush, J. S. et al. Themolecular mechanism of $\mathrm{N}$-acetylglucosamine side-chain attachment to the Lancefield group A carbohydrate in Streptococcus pyogenes.J. Biol. Chem. 292, 19441-19457 (2017).

16. Le Breton, Y. et al. Essential genes in the core genome of the human pathogen Streptococcus pyogenes. Sci. Rep. 5, 9838 (2015).

17. van Hensbergen, V. P. et al. Streptococcal Lancefield polysaccharides are critical cell wall determinants for human Group IIA secreted phospholipase A2 to exert its bactericidal effects. PLoS Pathog. 14, e1007348 (2018).

18. Weiss, J. P. Molecular determinants of bacterial sensitivity and resistance to mammalian Group IIA phospholipase A2. Biochim. Biophys. Acta 1848, 3072-3077 (2015).

19. Ong, C. L., GillenC. M., Barnett, T. C., Walker, M. J. \& McEwan, A. G. An antimicrobial role for zinc in innate immune defense against group A Streptococcus.J. Infect. Dis. 209, 1500-1508 (2014).

20. Graham, M. R. et al. Virulence control in group A Streptococcus by a two-component gene regulatorysystem: global expression profiling and in vivo infection modeling. Proc. Natl Acad. Sci. USA 99, 13855-13860 (2002).

21. Lu, D. et al. Structure-based mechanism of lipoteichoic acid synthesis by Staphylococcus aureus LtaS. Proc. Natl Acad. Sci. USA 106, 1584-1589 (2009).

22. Schirner, K., Marles-Wright, J., Lewis, R. J. \& Errington, J. Distinct and essential morphogenic functions for wall- and lipo-teichoic acids in Bacillus subtilis. EMBO J. 28, 830-842 (2009).

23. Campeotto, I. et al. Structural and mechanistic insight into the Listeria monocytogenes two-enzyme lipoteichoic acid synthesis system. J. Biol. Chem. 289, 28054-28069 (2014).

24. Fischer, W., Laine, R. A. \& Nakano, M. On the relationship between glycerophosphoglycolipids and lipoteichoic acids in Gram-positive bacteria.
II Structures of glycerophosphoglycolipids. Biochim. Biophys. Acta 528, 298-308 (1978).

25. Karatsa-Dodgson, M., Wormann, M. E. \& Grundling, A. In vitro analysis of the Staphylococcus aureus lipoteichoic acid synthase enzyme using fluorescently labeled lipids. J. Bacteriol. 192, 5341-5349 (2010).

26. Kennedy, E. P., Rumley, M. K., Schulman, H. \& Van Golde, L. M. Identification of $s n$-glycero-1-phosphate and phosphoethanolamine residues linked to the membrane-derived oligosaccharides of Escherichia coli. J. Biol. Chem. 251, 4208-4213 (1976).

27. Marino, J. P. et al. Three-dimensional triple-resonance ${ }^{1} \mathrm{H},{ }^{13} \mathrm{C},{ }^{31} \mathrm{P}$ experiment: sequential through-bond correlation of ribose protons and intervening phosphorus along the RNA oligonucleotide backbone. J. Am. Chem. Soc. 116, 6472-6473 (1994)

28. Heymann, H., Manniello, J. M. \& Barkulis, S. S. Structure of streptococcal cell walls. V. Phosphate esters in the walls of group A Streptococcus pyogenes. Biochem. Biophys. Res. Commun. 26, 486-491 (1967).

29. Emdur, L. I., Saralkar, C., McHugh, J. G. \& Chiu, T. H. Glycerolphosphatecontaining cell wall polysaccharides from Streptococcus sanguis. J. Bacteriol. 120, 724-732 (1974).

30. Prakobphol, A., Linzer, R. \& Genco, R. J. Purification and characterization of a rhamnose-containing cell wall antigen of Streptococcus mutans B13 (serotype d). Infect. Immun. 27, 150-157 (1980).

31. Pritchard, D. G., Michalek, S. M., McGhee, J. R. \& Furner, R. L. Structure of the serotype $\mathrm{f}$ polysaccharide antigen of Streptococcus mutans. Carbohydr. Res. 166, 123-131 (1987).

32. Pritchard, D. G., Gregory, R. L., Michalek, S. M. \& McGhee, J. R. Characterization of the serotype e polysaccharide antigen of Streptococcus mutans. Mol. Immunol. 23, 141-145 (1986).

33. Vickery, C. R., Wood, B. M., Morris, H. G., Losick, R. \& Walker, S. Reconstitution of Staphylococcus aureus lipoteichoic acid synthase activity identifies Congo red as a selective inhibitor. J. Am. Chem. Soc. 140, 876-879 (2018)

34. Neuhaus, F. C. \& Baddiley, J. A continuum of anionic charge: structures and functions of D-alanyl-teichoic acids in Gram-positive bacteria. Microbiol. Mol. Biol. Rev. 67, 686-723 (2003).

35. Peschel, A. et al. Inactivation of the dlt operon in Staphylococcus aureus confers sensitivity to defensins, protegrins, and other antimicrobial peptides. J. Biol. Chem. 274, 8405-8410 (1999).

36. Falagas, M. E., Rafailidis, P. I. \& Matthaiou, D. K. Resistance to polymyxins: mechanisms, frequency and treatment options. Drug Resist. Updat. 13, 132-138 (2010)

37. Djoko, K. Y., Ong, C. L., Walker, M. J. \& McEwan, A. G. The role of copper and zinc toxicity in innate immune defense against bacterial pathogens. J. Biol. Chem. 290, 18954-18961 (2015).

38. Ong, C. L., Walker, M. J. \& McEwan, A. G. Zinc disrupts central carbon metabolism and capsule biosynthesis in Streptococcus pyogenes. Sci. Rep. 5, 10799 (2015).

39. McDevitt, C. A. et al. A molecular mechanism for bacterial susceptibility to zinc. PLoS Pathog. 7, e1002357 (2011).

40. Buckland, A. G. \& Wilton, D. C. Inhibition of secreted phospholipases A2 by annexin V. Competition for anionic phospholipid interfaces allows an assessment of the relative interfacial affinities of secreted phospholipases A2. Biochim. Biophys. Acta 1391, 367-376 (1998).

41. Koprivnjak, T., Peschel, A., Gelb, M. H., Liang, N. S. \& Weiss, J. P. Role of charge properties of bacterial envelope in bactericidal action of human group IIA phospholipase A2 against Staphylococcus aureus. J. Biol. Chem. 277, 47636-47644 (2002).

42. Hunt, C. L., Nauseef, W. M. \& Weiss, J. P. Effect of d-alanylation of (lipo) teichoic acids of Staphylococcus aureus on host secretory phospholipase A2 action before and after phagocytosis by human neutrophils. J. Immunol. 176, 4987-4994 (2006).

43. Carapetis, J. R., Steer, A. C., Mulholland, E. K. \& Weber, M. The global burden of group A streptococcal diseases. Lancet Infect. Dis. 5, 685-694 (2005).

44. Goldstein, I., Rebeyrotte, P., Parlebas, J. \& Halpern, B. Isolation from heart valves of glycopeptides which share immunological properties with Streptococcus haemolyticus group A polysaccharides. Nature 219, 866-868 (1968).

45. Ayoub, E. M. \& Dudding, B. A. Streptococcal group A carbohydrate antibody in rheumatic and nonrheumatic bacterial endocarditis. J. Lab. Clin. Med. 76, 322-332 (1970).

46. Kirvan, C. A., Swedo, S. E., Heuser, J. S. \& Cunningham, M. W. Mimicry and autoantibody-mediated neuronal cell signaling in Sydenham chorea. Nat. Med. 9, 914-920 (2003).

47. Le Breton, Y. et al. Genome-wide discovery of novel M1T1 group A streptococcal determinants important for fitness and virulence during soft-tissue infection. PLoS Pathog. 13, e1006584 (2017).

48. Anders, S. \& Huber, W. Differential expression analysis for sequence count data. Genome Biol. 11, R106 (2010).

49. Robinson, M. D., McCarthy, D. J. \& Smyth, G. K. edgeR: a Bioconductor package for differential expression analysis of digital gene expression data. Bioinformatics 26, 139-140 (2010). 


\section{Acknowledgements}

This work was supported by the Center of Biomedical Research Excellence (COBRE) Pilot Grant (to N.K., K.V.K. and J.S.R.) supported by NIH grant No. P30GM110787 from the National Institute of General Medical Sciences (NIGMS); NIH grant No. R56AI135021 from the National Institute of Allergy and Infectious Diseases (NIAID) (to N.K.); VIDI grant No. 91713303 from the Netherlands Organization for Scientific Research (NWO) (to N.M.vS. and V.P.vH.); the Swedish Research Council (Nos. 20134859 and 2017-03703) and The Knut and Alice Wallenberg Foundation (to G.W.); NIH grant No. P30GM127211 from the NIGMS and NIH grant No. 1S10OD021753 (to A.J.M.); the National Health and Medical Research Council of Australia (to M.J.W.); grants from CNRS, ANR (MNaims No. ANR-17-CE17-0012-01) and FRM (No. SPF20150934219) (to G.L.); NIH grant No. AI047928 from NIAID (to K.S.M. and Y.L.B.); and NIH grant No. AI094773 (to N.M.E.S. and A.T.B.). Carbohydrate composition analysis at the Complex Carbohydrate Research Center was supported by the Chemical Sciences, Geosciences and Biosciences Division, Office of Basic Energy Sciences, US Department of Energy grant (No. DE-FG02-93ER20097) to P.A. Use of the Advanced Photon Source was supported by the US Department of Energy, Office of Science, Office of Basic Energy Sciences, under Contract No. W-31-109-Eng-38 and NIH grants Nos. S10_RR25528 and S10_RR028976. Use of the Stanford Synchrotron Radiation Lightsource, SLAC National Accelerator Laboratory, is supported by the US Department of Energy, Office of Science, Office of Basic Energy Sciences under Contract No. DE-AC02-76SF00515. The SSRL Structural Molecular Biology Program is supported by the DOE Office of Biological and Environmental Research, and by the NIH, NIGMS including No. P41GM103393. The contents of this publication are solely the responsibility of the authors and do not necessarily represent the official views of NIGMS or NIH.

\section{Author contributions}

A.R., P.D., Y.L.B., K.S.M., A.G.M., A.J.M., G.L., M.J.W., J.S.R., K.V.K., G.W., N.M.vS. and N.K. designed the experiments. R.J.E., V.P.vH., A.R., A.T., J.S.R., K.V.K., G.W. and N.K. performed functional and biochemical experiments. K.V.K. carried out X-ray crystallography and structure analysis. A.R. and G.W. performed NMR studies. P.D. and A.J.M. performed MS analysis. V.P.vH., N.K. and K.V.K. constructed plasmids and isolated mutants. R.J.E., V.P.vH., A.R., P.D., Y.L.B., N.M.E.S., A.T.B., K.S.M., A.G.M., A.J.M., M.J.W., J.S.R., K.V.K., G.W., N.M.vS. and N.K. analyzed the data. N.M.vS. and N.K. wrote the manuscript with contributions from all authors. All authors reviewed the results and approved the final version of the manuscript.

\section{Competing interests}

The authors declare no competing interests.

\section{Additional information}

Supplementary information is available for this paper at https://doi.org/10.1038/ s41589-019-0251-4.

Reprints and permissions information is available at www.nature.com/reprints. Correspondence and requests for materials should be addressed to N.M.v. or N.K.

Publisher's note: Springer Nature remains neutral with regard to jurisdictional claims in published maps and institutional affiliations.

(c) The Author(s), under exclusive licence to Springer Nature America, Inc. 2019 


\section{Methods}

Bacterial strains, growth conditions and media. All plasmids, strains and primers used in this study are listed in Supplementary Tables 5 and 6. GAS and S. mutans strains were grown in Todd-Hewitt broth supplemented with $1 \%$ yeast extract (THY) without aeration at $37^{\circ} \mathrm{C}$. S. mutans plates were grown with $5 \% \mathrm{CO}_{2}$. For hGIIA-mediated killing experiments, $S$. mutans strains were grown in ToddHewitt broth without yeast extract and with $5 \% \mathrm{CO}_{2}$. E. coli strains were grown in Lysogeny broth medium or on Lysogeny broth agar plates at $37^{\circ} \mathrm{C}$. When required, antibiotics were included at the following concentrations: ampicillin at $100 \mu \mathrm{g} \mathrm{ml}^{-1}$ for E. coli; streptomycin at $100 \mu \mathrm{g} \mathrm{ml}^{-1}$ for E. coli; erythromycin (Erm) at 500 $\mu \mathrm{g} \mathrm{ml}^{-1}$ for E. coli, $5 \mu \mathrm{g} \mathrm{ml}^{-1}$ for GAS and $10 \mu \mathrm{g} \mathrm{ml}^{-1}$ for S. mutans; chloramphenicol (CAT) at $10 \mu \mathrm{g} \mathrm{ml}^{-1}$ for E. coli and $2 \mu \mathrm{g} \mathrm{ml}^{-1}$ for GAS and S. mutans; spectinomycin at $200 \mu \mathrm{g} \mathrm{ml}^{-1}$ for E. coli, $100 \mu \mathrm{g} \mathrm{ml}^{-1}$ for GAS and $500 \mu \mathrm{g} \mathrm{ml}^{-1}$ for S. mutans; and kanamycin at $300 \mu \mathrm{g} \mathrm{ml}^{-1}$ for GAS

To identify genes providing resistance against $\mathrm{Zn}^{2+}$ toxicity, Roswell Park Memorial Institute (RPMI) 1640 (without glucose) (Gibco) was supplemented with guanine, adenine and uracil at a concentration of $25 \mu \mathrm{g} \mathrm{ml}^{-1}$ each, D-glucose at a concentration of $0.5 \% \mathrm{w} / \mathrm{v}$ and HEPES at $50 \mathrm{mM}$. Vitamins were provided by BME Vitamins 100× (Sigma, No. B6891).

Genetic manipulations. Plasmids were transformed into GAS and S. mutans by electroporation or natural transformation as described previously ${ }^{6}$. All constructs and mutants were confirmed by PCR and sequencing analysis (Eurofins MWG Operon and Macrogen).

Genetic manipulation of GAS: To construct 5005 $\Delta \mathrm{gacH}$ and $2221 \Delta \mathrm{gacH}$ 5005 chromosomal DNA was used as a template for amplification of two DNA fragments using primer pairs 5005-f/gacHdel-r and gacHdel-f/5005-r, which were fused and amplified using a PCR overlap method ${ }^{50}$ with primer pair $5005-\mathrm{f} / 5005-\mathrm{r}$ to create the deletion of $g a c H$. The PCR product was digested with BamHI and XhoI and ligated into BamHI/SalI-digested plasmid pBBL740, transformed into 5005 and 2221, and CAT-resistant colonies were selected on THY agar plates. Several potential double-crossover mutants were selected as previously described ${ }^{51}$

To construct the plasmid for in cis complementation of $5005 \mathrm{AgcH}, 5005$ chromosomal DNA was used as a template for amplification of $\mathrm{gacH}$ using the primer pair 5005-f/5005-r, which was cloned in pBBL740 through restrictionligation using BamHI and XhoI. The plasmid was transformed into the $5005 \Delta$ gacH strain, and CAT-resistant colonies were selected on THY agar plates. Double-crossover mutants were selected as described above.

To construct $5448 \Delta$ gacH, GAS 5448 chromosomal DNA was used to amplify up- and downstream regions flanking $\mathrm{gacH}$ using primer pairs 5448-f/5448CAT-r and 5448CAT-f/5448-r. Primers 5448CAT-f and 5448CAT-r contain 25 base pair (bp) extensions complementary to the CAT resistance cassette. Up- and downstream were fused to the CAT cassette using 5448-f/5448-r, and cloned into pHY304 through digestion-ligation using XhoI and HindIII, yielding plasmid pHY304 $\mathrm{gacH}$. After plasmid transformation into 5448, the double-crossover mutant $5448 \Delta$ gacH was selected as previously described ${ }^{15}$.

To complement $5448 \Delta$ gacH, $g a c H$ was amplified from 5448 chromosomal DNA using primer pair gacH-EcoRI-f/gacH-BglII-r, digested using EcoRI/BglII and ligated into EcoRI/BglII-digested pDCerm, yielding pgacH_erm. To make a catalytically inactive variant of $\mathrm{gacH}$, the mutation T550A was introduced into pgacH_erm using Gibson Assembly site-directed mutagenesis with the primers gacH-T530A-F, gacH-T530A-R, repB-isoF and repB-isoR. The plasmids were transformed into $5448 \mathrm{gacH}$ and selected for Erm resistance on THY agar plates. Transformation was confirmed by PCR, yielding strains $5448 \mathrm{gacH}$ :pgacH and 5448 gacH:pgacH-T530A, respectively.

To construct SMU $\Delta s c c H, S$. mutans $X c$ chromosomal DNA was used to amplify up- and downstream region flanking using primer pairs sccH-f/sccH-erm-r and sccH-erm-f /sccH-r. Primers sccH-erm-f and sccH-erm-r contained $25 \mathrm{bp}$ extensions complementary to the Erm resistance cassette. Up- and downstream PCR fragments were mixed with the Erm cassette and amplified as a single PCR fragment using primer pair $\mathrm{sccH}-\mathrm{f} / \mathrm{sccH}-\mathrm{r}$. The $s c \mathrm{H}$ knockout construct was transformed into S. mutans as described previously ${ }^{6}$. Erm-resistant single colonies were picked and checked for deletion of $s c \mathrm{H}$ and integration of Erm cassette by PCR, resulting in SMU $\Delta s c c H$. For complementation, $s c c H$ and $g a c H$ were amplified from $S$. mutans $X c$ and GAS 5448 chromosomal DNA, respectively, using primer pairs sccH-EcoRI-f/sccH-BglII-r and gacH-EcoRI-f/gacH-BglII-r. The PCR products were digested with EcoRI/BglII and ligated into EcoRI/BglII-digested pDC123 vector, yielding $\mathrm{psccH}$ and $\mathrm{pgacH}$ _cm, respectively. To make a catalytically inactive variant of $s c c H$, the mutation T553A was introduced into psccH using Gibson Assembly site-directed mutagenesis with the primers sccH-T553A-F, sccH$\mathrm{T} 553-\mathrm{R}$, repB-isoF and repB-isoR. The plasmids were transformed into SMU $\Delta s c H$ as previously described ${ }^{6}$. CAT-resistant single colonies were picked and checked for the presence of psccH or pgacH_cm by PCR, yielding strains SMU $\Delta s c c H$ :psccH, SMU $\Delta s c c H: p s c c H$-T533A and SMU $\Delta s c c H: p g a c H$, respectively.

To create a vector for expression of eGacH in E. coli, the gene was amplified from 5005 chromosomal DNA using the primers gacH-NcoI-f and gacH-XhoI-r. The PCR product was digested with $\mathrm{NcoI}$ and XhoI, and ligated into NcoI/XhoIdigested pCDF-NT vector. The resultant plasmid, pCDF-GacH, contained gacH fused at the $\mathrm{N}$ terminus with a His-tag followed by a TEV protease recognition site. To produce a catalytically inactive variant of $\mathrm{eGacH}$, the mutation T530A was introduced into pCDF-GacH using Gibson Assembly site-directed mutagenesis and the primers gacH-T530A-F, gacH-T530A-R, Str-isoF and Str-isoR.

\section{Identification of hGIIA-resistant GAS transposon mutants. The GAS M1T1} 5448 Krmit transposon mutant library ${ }^{16}$ was grown to mid-log phase $\left(\mathrm{OD}_{600}=0.4\right)$ Colony-forming units $\left(1 \times 10^{5}\right)$ were incubated with $27.5 \mu \mathrm{g} \mathrm{ml}^{-1}$ recombinant hGIIA ${ }^{52}$ in triplicate for $1 \mathrm{~h}$ at $37^{\circ} \mathrm{C}$ and plated on THY agar plates supplemented with kanamycin. The position of the transposon insertion of resistant colonies was determined as described previously ${ }^{53}$. hGIIA susceptibility experiments were performed as described previously ${ }^{17}$.

Determination of selective metal concentrations. To determine the target concentration of $\mathrm{Zn}^{2+}$, colonies of 5448 wild type and $5448 \Delta c z c D^{19}$ were scraped from THY agar plates, resuspended and washed in phosphate buffered saline (PBS) to $\mathrm{OD}_{600}=1$ and used to inoculate freshly prepared modified RPMI (mRPMI) containing varying concentrations of $\mathrm{Zn}^{2+}$ to $\mathrm{OD}_{600}=0.05$ in a 96-well plate. Growth at $37^{\circ} \mathrm{C}$ was monitored at $\mathrm{OD}_{595}$ every 15 min using the BMG Fluostar plate reader.

Tn-seq library screen for $\mathbf{Z n}^{2+}$ sensitivity. The $5448 \mathrm{Krmit} \mathrm{Tn}$-seq library at $\mathrm{T}_{0}$ generation ${ }^{16}$ was thawed, inoculated into $150 \mathrm{ml}$ prewarmed THY broth containing kanamycin and grown at $37^{\circ} \mathrm{C}$ for $6 \mathrm{~h}$. The culture $\left(\mathrm{T}_{1}\right)$ was centrifuged at $4,000 \mathrm{~g}$ for $15 \mathrm{~min}$ at $4^{\circ} \mathrm{C}$ and the pellet resuspended in $32.5 \mathrm{ml}$ saline. Freshly prepared

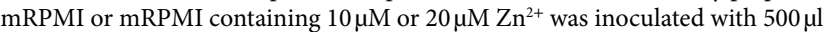
culture into $39.5 \mathrm{ml}$ media, creating a 1:20-fold inoculation. These $\mathrm{T}_{2}$ cultures were then grown at $37^{\circ} \mathrm{C}$ for exactly $6 \mathrm{~h}$, at which point $2 \mathrm{ml}$ of these cultures was inoculated again into $38 \mathrm{ml}$ of freshly prepared mRPMI alone or mRPMI containing $10 \mu \mathrm{M}$ or $20 \mu \mathrm{M} \mathrm{Zn}{ }^{2+}$. The remaining $38 \mathrm{ml}$ of $\mathrm{T}_{2}$ culture was harvested by centrifugation at $4,000 \mathrm{~g}$ for $10 \mathrm{~min}$ at $4{ }^{\circ} \mathrm{C}$ and pellets stored at $-20^{\circ} \mathrm{C}$ for subsequent DNA extraction. Cultures were grown for additional $6 \mathrm{~h}$, at which point $\mathrm{T}_{3}$ cultures were harvested by centrifugation at $4,000 \mathrm{~g}$ for $10 \mathrm{~min}$ at $4^{\circ} \mathrm{C}$ and pellets stored at $-20^{\circ} \mathrm{C}$.

Tn-seq Krmit transposon insertion tags were prepared from the cell pellets as previously described ${ }^{16,47}$. After quality control with the Bioanalyzer instrument (Agilent), the libraries of Krmit insertion tags were sequenced (50 nt single-end reads) on an Illumina HiSeq 1500 in the Institute for Bioscience and Biotechnology Research Sequencing Core at the University of Maryland, College Park. Tn-seq read datasets were analyzed (quality, filtering, trimming, alignment, visualization) as previously described ${ }^{16,47}$ using the M1T1 5448 genome as reference for read alignments. The ratios of mutant abundance comparing the output to input mutant pools were calculated as a fold change for each GAS gene using the DEseq 2 and EdgeR pipelines ${ }^{47-49}$.

Drop-test assays. Strains 5448 wild type, 5448 $\mathrm{gacI}, 5448 \Delta \mathrm{gacI}: \mathrm{gacI}, 5448 \Delta \mathrm{gacH}$, $5448 \Delta g a c H: p g a c H$, S. mutans wild type, SMU $\Delta s c c H$, SMU $\Delta s c c H: p s c c H$ and SMU $\Delta s c H$ :pgacH were grown in THY to mid-exponential growth phase, adjusted to $\mathrm{OD}_{600}=0.6$ and serially diluted. Five microliters were spotted onto THY agar plates containing varying concentrations of $\mathrm{Zn}^{2+}\left(\mathrm{ZnSO}_{4} \cdot 7 \mathrm{H}_{2} \mathrm{O}\right)$. Plates were incubated at $37^{\circ} \mathrm{C}$ overnight and photographed.

Protein expression and purification. To purify eGacH and eGacH-T530A, E. coli Rosetta(DE3) carrying the respective plasmid was grown in Lysogeny broth at $37^{\circ} \mathrm{C}$ to $\mathrm{OD}_{600}=0.4-0.6$ and induced with $0.25 \mathrm{mM}$ isopropyl $\beta-\mathrm{D}-1-$ thiogalactopyranoside (IPTG) at $18^{\circ} \mathrm{C}$ for approximately $16 \mathrm{~h}$. Bacteria were lysed in $20 \mathrm{mM}$ Tris- $\mathrm{HCl} \mathrm{pH} 7.5,300 \mathrm{mM} \mathrm{NaCl}$ by a microfluidizer cell disrupter. The soluble fractions were purified by nickel-nitrilotriacetic acid chromatography. The eluate was dialyzed into $20 \mathrm{mM}$ Tris- $\mathrm{HCl} \mathrm{pH} \mathrm{7.5,300} \mathrm{mM} \mathrm{NaCl}$ in the presence

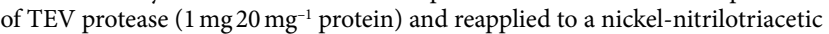
acid column equilibrated in $20 \mathrm{mM}$ Tris- $\mathrm{HCl} \mathrm{pH} \mathrm{7.5,300} \mathrm{mM} \mathrm{NaCl}$ to remove the cleaved His-tag and any uncleaved protein from the sample. The protein was further purified by size-exclusion chromatography on a Superdex 200 column in $20 \mathrm{mM}$ HEPES pH 7.5, $100 \mathrm{mM} \mathrm{NaCl}$.

To express seleno-methionine (Se-Met)-labeled eGacH, E. coli Rosetta(DE3) carrying eGacH was grown in Lysogeny broth at $37^{\circ} \mathrm{C}$ to $\mathrm{OD}_{600}=0.5$. The bacteria were centrifuged and resuspended in M9 minimal media supplemented with Se-Met. Protein expression was induced with $0.25 \mathrm{mM}$ IPTG, and the cultures were grown at $16^{\circ} \mathrm{C}$ for approximately $16 \mathrm{~h}$. Se-Met-labeled eGacH was purified as described above.

Crystallization, data collection and structure solution. The conditions for eGacH crystallization were initially screened using the JCSG Suites I-IV screens (Qiagen) at a protein concentration of $9 \mathrm{mg} \mathrm{ml}^{-1}$ by the hanging-drop vapor diffusion method. Crystals of Se-Met-substituted eGacH were grown in $0.1 \mathrm{M}$ HEPES pH 7.5, 10\% PEG8000 and 8\% ethylene glycol. Crystals were transferred to crystallization solution supplemented with $20 \%$ ethylene glycol and flash-frozen in liquid nitrogen. The data were collected at APS 22-ID at a wavelength of $0.9793 \AA$. Crystals of GroP•eGacH complex were obtained using crystallization solution containing $0.2 \mathrm{M}$ calcium acetate, 0.1 M MES pH 6.0 and 20\% PEG8000. 
sn-Gro-1-P (Sigma Aldrich) was mixed with eGacH at $10 \mathrm{mM}$ before crystallization. Initial crystals of the GroP•eGacH complex belonged to the same crystal form as apo $\mathrm{GacH}$, but crystals of different morphology grew epitaxially after several days. These crystals displayed better diffraction and were used for structure determination of the GroP•eGacH complex. Crystals were cryoprotected in crystallization solution supplemented with $10 \mathrm{mM}$ sn-glycerol-1-phosphate and $20 \%$ ethylene glycol and vitrified in liquid nitrogen. The data were collected at SSRL BL9-2 at a wavelength of $0.97946 \AA$.

All data were processed and scaled using XDS and XSCALE ${ }^{54}$. The structure of eGacH was solved by the Se single-wavelength anomalous diffraction method. Se atom positions were determined using the HySS module in $P H E N I X^{55}$. The structure was solved using AutoSol wizard in $P H E N I X^{55}$. The model was completed using Coot $^{56}$ and refined using phenix.refine in PHENIX ${ }^{55}$. Ramachandran distribution analysis of the $\mathrm{eGacH}$ final structure with MolProbity ${ }^{57}$ indicates that 96.6 and $3.4 \%$ residues are in favored and allowed regions, respectively, with no outliers.

The structure of the GroP•eGacH complex was solved by molecular replacement using Phaser in PHENIX ${ }^{55}$ and the dimer of apo eGacH as a search model. The model was adjusted using Coot and refined using phenix.refine. Difference electron density corresponding to GroP molecules was readily identified after refinement. GroP molecules were modeled using Coot. The geometric restraints for GroP were generated using Grade Web Server (http://grade.globalphasing.org) (Global Phasing). The last several rounds of refinement were performed using 19 translation/ libration/screw groups, which were identified by $P H E N I X^{55}$. Ramachandran distribution analysis of the $\mathrm{GroP} \bullet \mathrm{eacH}$ final structure indicated that 97.2 and $2.8 \%$ residues are in favored and allowed regions, respectively, with no outliers. The structures were validated using Coot, MolProbity and wwPDB Validation Service (https://validate.wwpdb.org). Statistics for data collection, refinement and model quality are listed in Supplementary Table 7.

In vitro assay of eGacH enzymatic activity. Purification of 16:0-6:0 NBDphosphatidylglycerol lipid (Avanti) was performed by preparative thin-layer chromatography as described previously ${ }^{25}$, dissolved in $\mathrm{CH}_{3} \mathrm{OH}$ and stored at $-20^{\circ} \mathrm{C}$ until use. The lipid was dried and dispersed in octyl-glucoside by sonication before addition of the remaining components. Reaction mixtures contained $0.05 \mathrm{M}$ sodium succinate $\mathrm{pH} 6.3,10 \mathrm{mM} \mathrm{MnCl}_{2}, 0.05 \mathrm{M} \mathrm{NaCl}, 0.25 \%$ octyl-glucoside, $20 \mu \mathrm{g}$ NBD-phosphatidylglycerol, ultrasonically dispersed in $0.5 \%$ octyl-glucoside (Branson 2200 bath sonicator) and either no enzyme, $20 \mu \mathrm{g} \mathrm{eGacH}$ or $20 \mu \mathrm{g} \mathrm{eGacH}-\mathrm{T} 530 \mathrm{~A}$ in a total volume of $0.02 \mathrm{ml}$. Following incubation at $37^{\circ} \mathrm{C}$ for $3 \mathrm{~h}$, the reaction was stopped by the addition of $0.08 \mathrm{ml} \mathrm{CHCl} / \mathrm{CH}_{3} \mathrm{OH}(2: 1)$ and analyzed for fluorescence on a BioRad ChemiDoc MP Imaging System using the fluorescein preset mode, as described previously ${ }^{25}$. The migration position of the NBD-diacylglycerol product was determined from the product of a separate reaction containing purified phospholipase $\mathrm{C}$ from B. cereus (Sigma Aldrich).

Isolation of cell wall. Cell wall was isolated from exponential phase cultures by the sodium dodecyl sulfate-boiling procedure and lyophilized as previously described ${ }^{15}$.

GAC purification. GAC was released from the cell wall by sequential digestion with mutanolysin (Sigma Aldrich) and recombinant PlyC amidase $\mathrm{a}^{15}$, and partially purified by a combination of size-exclusion chromatography and ionexchange chromatography. Mutanolysin digests contained $5 \mathrm{mg} \mathrm{ml}^{-1}$ of cell wall suspension in $0.1 \mathrm{M}$ sodium acetate, $\mathrm{pH} 5.5,2 \mathrm{mM} \mathrm{CaCl}_{2}$ and $5 \mathrm{U} \mathrm{ml}^{-1}$ mutanolysin Following overnight incubation at $37^{\circ} \mathrm{C}, \mathrm{GAC}$ was separated from the cell wall by centrifugation at $13,000 \mathrm{~g}, 10 \mathrm{~min}$ and precipitated from $80 \%$ acetone $\left(-20^{\circ} \mathrm{C}\right)$. The precipitate was sedimented $(5,000 \mathrm{~g}, 20 \mathrm{~min})$, dried briefly under nitrogen gas and redissolved in $0.1 \mathrm{M}$ Tris- $\mathrm{HCl}, \mathrm{pH} 7.4$ and digested with PlyC $\left(50 \mu \mathrm{g} \mathrm{ml}^{-1}\right)$ overnight at $37^{\circ} \mathrm{C}$. Following $\mathrm{PlyC}$ digestion, GAC was recovered by acetone precipitation as described above, redissolved in a small volume of $0.2 \mathrm{~N}$ acetic acid and chromatographed on a $25 \mathrm{ml}$ column of BioGel P10 equilibrated in $0.2 \mathrm{~N}$ acetic acid. Fractions $(1.5 \mathrm{ml})$ were collected and monitored for carbohydrate by the anthrone assay. Fractions containing GAC were combined, concentrated and desalted by spin column centrifugation (3,000 MW cutoff filter). GAC was loaded onto an $18 \mathrm{ml}$ column of DEAE-Sephacel. The column was eluted with a $100 \mathrm{ml}$ gradient of $\mathrm{NaCl}(0-1 \mathrm{M})$. Fractions were analyzed for carbohydrate by the anthrone assay and phosphate by the malachite green assay following digestion with $70 \%$ perchloric acid. Fractions containing peaks of carbohydrate were combined, concentrated by spin column (3,000 MW cutoff) and lyophilized.

Anthrone assay. Total carbohydrate content was determined by a minor modification of the anthrone procedure. Reactions contained $0.08 \mathrm{ml}$ of aqueous sample and water and $0.32 \mathrm{ml}$ anthrone reagent $(0.2 \%$ anthrone in concentrated $\mathrm{H}_{2} \mathrm{SO}_{4}$ ). The samples were heated to $100^{\circ} \mathrm{C}$ for $10 \mathrm{~min}$, cooled in water (room temperature) and the absorbance at $580 \mathrm{~nm}$ was recorded. GAC concentration was estimated using an L-rhamnose standard curve.

Phosphate assay. Approximately $1.5 \mathrm{mg}$ of GAS cell wall material was dissolved in $400 \mu \mathrm{l} \mathrm{H}_{2} \mathrm{O}$ and $8 \mu \mathrm{g} \mathrm{ml}^{-1} \mathrm{PlyC}$ and incubated at $37^{\circ} \mathrm{C}$, rotating for approximately $16 \mathrm{~h}$. Additional PlyC was added and incubated for a further $4-6 \mathrm{~h}$. To liberate SCC from S. mutans cell walls, $1.5 \mathrm{mg}$ of $S$. mutans cell wall material was incubated
$24 \mathrm{~h}$ with $1.5 \mathrm{U} \mathrm{ml}^{-1}$ mutanolysin in $400 \mu \mathrm{l}$ of $0.1 \mathrm{M}$ sodium acetate, $\mathrm{pH} 5.5$ and $2 \mathrm{mM} \mathrm{CaCl}_{2}$. The samples were incubated at $100^{\circ} \mathrm{C}$ for $20 \mathrm{~min}$ and centrifuged for $5 \mathrm{~min}$ at maximum speed in a table top centrifuge. The supernatant was transferred to a new micro-centrifuge tube and incubated with $2 \mathrm{~N} \mathrm{HCl}$ at $100^{\circ} \mathrm{C}$ for $2 \mathrm{~h}$. The samples were neutralized with $\mathrm{NaOH}$ in the presence of $62.5 \mathrm{mM}$ HEPES pH 7.5. To $100 \mu \mathrm{l}$ of acid hydrolyzed sample, $2 \mu \mathrm{l}$ of $1 \mathrm{U}_{\mu l^{-1}}$ alkaline phosphatase (Thermo Fisher) and $10 \mu \mathrm{l} 10 \times$ alkaline phosphatase buffer was added and incubated at $37^{\circ} \mathrm{C}$, rotating, overnight. Released phosphate was measured using the PiColorLock Gold kit (Innova Biosciences), according to the manufacturer's protocol.

Total phosphate content was determined by the malachite green method following digestion with perchloric acid. Samples containing $10-80 \mu \mathrm{l}$ were heated to $110^{\circ} \mathrm{C}$ with $40 \mu \mathrm{l} 70 \%$ perchloric acid (Fisher Scientific) in $13 \times 100$ borosilicate disposable culture tubes for $1 \mathrm{~h}$. The reactions were diluted to $160 \mu \mathrm{l}$ with water and $100 \mu \mathrm{l}$ was transferred to a flat-bottom 96-well culture plate. Malachite green reagent $(0.2 \mathrm{ml})$ was added and the absorbance at $620 \mathrm{~nm}$ was read after $10 \mathrm{~min}$ at room temperature. Malachite green reagent contained 1 vol. $4.2 \%$ ammonium molybdate tetrahydrate (by weight) in $4 \mathrm{M} \mathrm{HCl}, 3$ vol. $0.045 \%$ malachite green (by weight) in water and $0.01 \%$ Tween 20 .

Glycerol assay. Samples for glycerol measurement were prepared as described for the phosphate assay but were not digested with alkaline phosphatase. Instead, glycerol concentration was measured using the Glycerol Colorimetric assay kit (Cayman Chemical) according to the manufacturer's protocol.

Glycosyl composition analysis. Glycosyl composition analysis of GAS and S. mutans cell wall samples was performed at the Complex Carbohydrate Research Center by combined gas chromatography/mass spectrometry of the per-Otrimethylsilyl derivatives of the monosaccharide methyl glycosides produced from the sample by acidic methanolysis as described previously ${ }^{15}$.

Dot-blot analysis of GAC. The semi-quantitative immuno-dot-blot analysis of GAC expressed by GAS strains was conducted essentially as described ${ }^{15}$ with the following minor modification: the PlyC-digested cell wall fractions were serially diluted before spotting to a nitrocellulose membrane.

Total phospholipid content analysis. GAS cells $(50 \mathrm{ml})$ grown in THY broth to $\mathrm{OD}_{600}=0.5$ were centrifuged, washed with PBS, resuspended in $5 \mathrm{ml} \mathrm{PBS}$ and incubated with PlyC $\left(100 \mu \mathrm{g} \mathrm{ml}^{-1}\right)$ at $37^{\circ} \mathrm{C}$ for $1 \mathrm{~h}$. A total lipid extract was prepared by a modification of Bligh-Dyer extraction, freed of non-lipid contaminants by Folch partitioning, as described previously ${ }^{15}$, and redissolved in $1 \mathrm{ml} \mathrm{CHCl}_{3} /$ $\mathrm{CH}_{3} \mathrm{OH}(2: 1)$. An aliquot $(20 \mu \mathrm{l})$ was analyzed for total phospholipid using the malachite green phosphate assay following perchloric acid digestion at $120^{\circ} \mathrm{C}$ for $1 \mathrm{~h}$, and the remainder was analyzed for phosphatidylglycerol.

Phosphatidylglycerol and NBD-diacylglycerol analysis. The analysis of phosphatidylglycerol and NBD-diacylglycerol was performed using an Ultimate 3000 ultra HPLC system coupled to a Thermo Q-Exactive Orbitrap mass spectrometer equipped with a heated electrospray ion source (Thermo Scientific). Lipid extracts were separated on a Waters ACQUITY BEH C8 column $(2.1 \times 100 \mathrm{~mm}, 1.7 \mu \mathrm{m})$ with the temperature maintained at $40^{\circ} \mathrm{C}$. The flow rate was $250 \mu \mathrm{min}^{-1}$, and the mobile phases consisted of 60:40 water/acetonitrile (A) and 90:10 isopropanol/acetonitrile (B), both containing $10 \mathrm{mM}$ ammonium formate and $0.1 \%$ formic acid. The samples were eluted with a linear gradient from $32 \% \mathrm{~B}$ to $97 \% \mathrm{~B}$ over $25 \mathrm{~min}$, maintained at $97 \% \mathrm{~B}$ for $4 \mathrm{~min}$ and re-equilibration with $32 \% \mathrm{~B}$ for $6 \mathrm{~min}$. The sample injection volume was $5 \mu \mathrm{l}$. The mass spectrometer was operated in positive and negative ionization modes. The full-scan and fragment spectra were collected at a resolution of 70,000 and 17,500, respectively. Data analysis and lipid identification were performed using Xcalibur 4.0 and Lipidsearch 4.1 (Thermo Fisher).

Total carbohydrate content analysis. GAS cells $(10 \mathrm{ml})$ grown in THY broth $\mathrm{OD}_{600}=0.5$ were centrifuged, washed twice with PBS and resuspended in $0.2 \mathrm{ml}$ distilled water. The cell suspension was assayed for carbohydrate content by the anthrone assay as described above.

Analysis of GAC for GroP. GAC (prepared from 1.5 mg of cell wall) was hydrolyzed in $0.1 \mathrm{ml} 0.5 \mathrm{M} \mathrm{NaOH}\left(100^{\circ} \mathrm{C}, 1 \mathrm{~h}\right)$ to release GroP, as described in ref. ${ }^{26}$. Following alkaline treatment, the reaction was neutralized with acetic acid, supplemented with $5 \mathrm{nmol}$ of citronellyl phosphate (as internal standard) and centrifuged on an Amicon Ultra Centrifugal Filter ( $0.5 \mathrm{ml}, 3,000$ nominal molecular weight limit). The filtrate was lyophilized and analyzed with a Q-Exactive mass spectrometer and an Ultimate 3000 ultra HPLC system (Thermo Fisher Scientific) using a silica-based SeQuant ZIC-pHILIC column $(2.1 \times 150 \mathrm{~mm}$, $5 \mu \mathrm{m}$, Merck) with elution buffers consisting of (1) $20 \mathrm{mM}\left(\mathrm{NH}_{4}\right)_{2} \mathrm{CO}_{3}$ with $0.1 \% \mathrm{NH}_{4} \mathrm{OH}$ in $\mathrm{H}_{2} \mathrm{O}$ and (2) acetonitrile. The column temperature was maintained at $40{ }^{\circ} \mathrm{C}$, and the flow rate was set to $150 \mu \mathrm{min}^{-1}$. MS detection was performed by electrospray ionization in negative ionization mode with source voltage maintained at $3.0 \mathrm{kV}$. The capillary temperature, sheath gas flow and auxiliary gas flow were set 
at $275^{\circ} \mathrm{C}, 40$ arb and 15 arb units, respectively. Full-scan MS spectra (mass range $m / z 75-1,000$ ) were acquired with resolution $R=70,000$ and AGC target $1 \times 10^{6}$. Extracted ion chromatograms for GroP and citronellyl phosphate were obtained from the LC-MS chromatograms and used to estimate relative GroP content.

Identification of the stereochemistry of the GroP moiety of GAC. GroP was liberated from GAC by alkaline hydrolysis as described in ref. ${ }^{26}$ and re-fractionated on BioGel P10. The bulk of the GAC elutes in the void volume and GroP elutes in the inclusion volume as identified by LC-MS. Column fractions containing GroP were combined, concentrated by rotary evaporation $\left(30^{\circ} \mathrm{C}\right.$, under reduced pressure) and desalted on BioGel P2. The stereochemistry of the GroP was determined by an enzymatic method using the Amplite Fluorimetric sn-Gro-3-P Assay Kit (AAT Bioquest) according to the manufacturer's instructions.

NMR spectroscopy. The NMR spectra were recorded on a Bruker AVANCE III $700 \mathrm{MHz}$ equipped with a $5 \mathrm{~mm}$ TCI Z-Gradient Cryoprobe $\left({ }^{1} \mathrm{H} /{ }^{13} \mathrm{C} /{ }^{15} \mathrm{~N}\right)$ and dual receivers, and a Bruker AVANCE II $600 \mathrm{MHz}$ spectrometer equipped with a $5 \mathrm{~mm}$ TXI inverse Z-Gradient ${ }^{1} \mathrm{H} / \mathrm{D}-{ }^{31} \mathrm{P} /{ }^{13} \mathrm{C}$. The ${ }^{1} \mathrm{H}$ and ${ }^{13} \mathrm{C}$ NMR chemical shift assignments of the polysaccharide material were carried out in $\mathrm{D}_{2} \mathrm{O}$ solution $(99.96 \%)$ at $323.2 \mathrm{~K}$ unless otherwise stated. Chemical shifts are reported in parts per million using internal sodium 3-trimethylsilyl- $\left(2,2,3,3-{ }^{2} \mathrm{H}_{4}\right)$-propanoate (TSP, $\left.\delta_{\mathrm{H}} 0.00 \mathrm{ppm}\right)$, external 1,4-dioxane in $\mathrm{D}_{2} \mathrm{O}\left(\delta_{\mathrm{C}} 67.40 \mathrm{ppm}\right)$ and $2 \% \mathrm{H}_{3} \mathrm{PO}_{4}$ in $\mathrm{D}_{2} \mathrm{O}\left(\delta_{\mathrm{p}} 0.00 \mathrm{ppm}\right)$ as reference. The ${ }^{1} \mathrm{H},{ }^{1} \mathrm{H}$-TOCSY experiments (dipsi2ph) were recorded with mixing times of $10,30,60,90$ and $120 \mathrm{~ms}$. The ${ }^{1} \mathrm{H},{ }^{1} \mathrm{H}-\mathrm{NOESY}$ experiments ${ }^{58}$ were collected with mixing times of 100 and $200 \mathrm{~ms}$. Uniform and non-uniform sampling (50 and 25\% NUS) were used for the multiplicity-edited ${ }^{1} \mathrm{H},{ }^{13} \mathrm{C}$-HSQC experiments ${ }^{59}$ employing an echo/anti-echo-TPPI gradient selection with and without decoupling during the acquisition. The $2 \mathrm{D}{ }^{1} \mathrm{H},{ }^{13} \mathrm{C}-\mathrm{HSQC}$-TOCSY experiments were acquired using MLEV17 for homonuclear Hartman-Hahn mixing, an echo/anti-echo-TPPI gradient selection with decoupling during acquisition and mixing times of $20,40,80$ and $120 \mathrm{~ms}$. The $2 \mathrm{D}{ }^{1} \mathrm{H},{ }^{31} \mathrm{P}-\mathrm{Hetero}$ TOCSY experiments ${ }^{60}$ were collected using a DIPSI 2 sequence with mixing times of $10,20,30,50$ and $80 \mathrm{~ms}$. The $2 \mathrm{D}{ }^{1} \mathrm{H},{ }^{31} \mathrm{P}-\mathrm{HMBC}$ experiments were recorded using an echo/anti-echo gradient selection and mixing times of 25, 50 and $90 \mathrm{~ms}$. The $3 \mathrm{D}^{1} \mathrm{H},{ }^{13} \mathrm{C},{ }^{31} \mathrm{P}^{27}$ spectra were obtained using echo/anti-echo gradient selection and constant time in $t_{2}$ with a nominal value of ${ }^{\mathrm{n}} J_{\mathrm{CP}}$ of $5 \mathrm{~Hz}$ and without multiplicity selection. The spectra were processed and analyzed using TopSpin 4.0.1 software (Bruker BioSpin).

Statistical analysis. Unless otherwise indicated, statistical analysis was carried out on pooled data from at least three independent biological repeats. A two-way ANOVA with Bonferroni multiple comparison test was used to compare multiple groups. A $P$ value equal to or less that 0.05 was considered statistically significant.
Reporting Summary. Further information on research design is available in the Nature Research Reporting Summary linked to this article.

\section{Data availability}

Illumina sequencing reads from the Tn-seq analysis were deposited in the NCBI Sequence Read Archive under the accession number SRP150081. The Tn-seq data, analyses and pipeline for the Tn-seq analyses are accessible under the DOI number 10.5281/zenodo.2541163 in GitHub as the following link: https://doi.org/10.5281/ zenodo.2541163. Atomic coordinates and structure factors of the reported crystal structures have been deposited in the Protein Data Bank with accession codes $5 \mathrm{U} 9 \mathrm{Z}$ (apo eGacH) and 6DGM (GroP•eGacH complex). All data generated during this study are included in the article, and supplementary information files or will be available from the corresponding authors upon reasonable request.

\section{References}

50. Horton, R. M., Hunt, H. D., Ho, S. N., Pullen, J. K. \& Pease, L. R. Engineering hybrid genes without the use of restriction enzymes: gene splicing by overlap extension. Gene 77, 61-68 (1989).

51. Trevino, J., LiuZ., Cao, T. N., Ramirez-Pena, E. \& Sumby, P. RivR is a negative regulator of virulencefactor expression in group A Streptococcus. Infect. Immun. 81, 364-372 (2013).

52. Ghomashchi, F. et al. Preparation of the full set of recombinant mouse- and human-secreted phospholipases A2. Methods Enzymol. 583, 35-69 (2017).

53. Le Breton, Y. \& McIver, K. S. Genetic manipulation of Streptococcus pyogenes (the group A Streptococcus, GAS). Curr. Protoc. Microbiol. 30, Unit 9D 3 (2013).

54. Kabsch, W. Xds. Acta Crystallogr. 66, 125-132 (2010).

55. Adams, P. D. et al. PHENIX: a comprehensive Python-based system for macromolecular structure solution. Acta Crystallogr. 66, 213-221 (2010).

56. Emsley, P., Lohkamp, B., Scott, W. G. \& Cowtan, K. Features and development of Coot. Acta Crystallogr. 66, 486-501 (2010).

57. Chen, V. B. et al. MolProbity: all-atom structure validation for macromolecular crystallography. Acta Crystallogr. 66, 12-21 (2010).

58. Wagner, R. \& Berger, S. Gradient-selected NOESY-A fourfold reduction of the measurement time for the NOESY Experiment. J. Magn. Reson. 123, 119-121 (1996).

59. Willker, W., Leibfritz, D., Kerssebaum, R. \& Bermel, W. Gradient selection in inverse heteronuclear correlation spectroscopy. Magn. Reson. Chem. 31, 287-292 (1993)

60. Kellogg, G. W. Proton-detected hetero-TOCSY experiments with application to nucleic acids. J. Magn. Reson. 98, 176-182 (1992). 


\section{Reporting Summary}

Nature Research wishes to improve the reproducibility of the work that we publish. This form provides structure for consistency and transparency in reporting. For further information on Nature Research policies, see Authors \& Referees and the Editorial Policy Checklist.

\section{Statistical parameters}

When statistical analyses are reported, confirm that the following items are present in the relevant location (e.g. figure legend, table legend, main text, or Methods section).

n/a Confirmed

$\bigotimes$ The exact sample size $(n)$ for each experimental group/condition, given as a discrete number and unit of measurement

$\searrow$ An indication of whether measurements were taken from distinct samples or whether the same sample was measured repeatedly

$\triangle$ The statistical test(s) used AND whether they are one- or two-sided

$\triangle$ Only common tests should be described solely by name; describe more complex techniques in the Methods section.

Х $\square$ A description of all covariates tested

$\square$ A description of any assumptions or corrections, such as tests of normality and adjustment for multiple comparisons

$\square \bigotimes$ A full description of the statistics including central tendency (e.g. means) or other basic estimates (e.g. regression coefficient) AND variation (e.g. standard deviation) or associated estimates of uncertainty (e.g. confidence intervals)

$\varnothing$ For null hypothesis testing, the test statistic (e.g. $F, t, r$ ) with confidence intervals, effect sizes, degrees of freedom and $P$ value noted

Give $P$ values as exact values whenever suitable.

Х $\square$ For Bayesian analysis, information on the choice of priors and Markov chain Monte Carlo settings

Х $\square$ For hierarchical and complex designs, identification of the appropriate level for tests and full reporting of outcomes

Х $\square$ Estimates of effect sizes (e.g. Cohen's $d$, Pearson's $r$ ), indicating how they were calculated

Clearly defined error bars

$\bigotimes$ State explicitly what error bars represent (e.g. SD, SE, CI)

Our web collection on statistics for biologists may be useful.

\section{Software and code}

\section{Policy information about availability of computer code}

Data collection Macromolecular structural data were collected using Sergui (SER-CAT, APS, beamline 22-ID) and Blu-Ice 5 (SSRL, beamline 9-2). The data
were processed using XDS and XSCALE, versions May 1, 2016 BUILT=20160617 and Jan 26, 2018, BUILT=20180126.
Mass-spectrometry data were collected using Thermo Xcalibur 4.0 (Thermo Fisher Scientific, Inc.).
The sequences of GacH, LtaS, and LtaP for construction of the phylogenetic tree were obtained from the ncbi database using blastp 2.7.1.
The NMR spectra were recorded on a Bruker AVANCE III $700 \mathrm{MHz}$ equipped with a $5 \mathrm{~mm} \mathrm{TCI} \mathrm{Z-Gradient} \mathrm{Cryoprobe} \mathrm{(1H/13C/15N)} \mathrm{and}$
dual receivers and a Bruker AVANCE II $600 \mathrm{MHz}$ spectrometer equipped with a $5 \mathrm{~mm} \mathrm{TXI} \mathrm{inverse} \mathrm{Z-Gradient} \mathrm{1H/D-31P/13C.} \mathrm{The} \mathrm{spectra}$
were acquired using TopSpin 3.1 software (Bruker BioSpin) for the $600 \mathrm{MHz}$ machine and TopSpin 3.2 (Bruker BioSpin) for the $700 \mathrm{MHz}$
machine. 

Validation Service rb-20031172, Lipidsearch 4.1, TOPCONS 2.0, HHpred (May 19, 2017), TMHMM 2.0.

Tn-seq data were analyzed using Bioperl: 1.7.1, Biopieces: 2.0, Cutadapt: 1.13, DESeq2: 1.20.0, EdgeR: 3.22.3, hpgltools (version 2018.03), commit id: c730ef178f8e57bbf3819e21cf5e6cfe879e6328, limma: 3.36.2.

Macromolecular structural data were analyzed using PHENIX and phenix.refine, versions dev_2481 and dev_3139; Phaser, version 2.8.2; Coot, version 0.8.9.1. Mass-spectrometry data were analyzed using Thermo Xcalibur 4.0 (Thermo Fisher Scientific, Inc.).

Phylogenetic analysis was done using MEGA6 6.0 and MUSCLE 3.5. The NMR spectra were processed, analyzed and plotted using TopSpin 4.0.1 software (Bruker BioSpin).

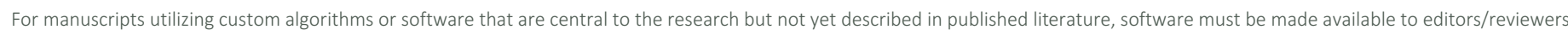

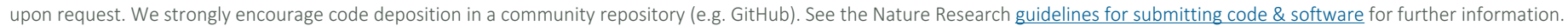

\section{Data}

Policy information about availability of data

All manuscripts must include a data availability statement. This statement should provide the following information, where applicable:

- Accession codes, unique identifiers, or web links for publicly available datasets

- A list of figures that have associated raw data

- A description of any restrictions on data availability

The structure factors and coordinates were deposited to the Protein Data Bank and released with accession codes 5U9Z (apo eGacH) and 6DGM (GroP•eGacH complex).

Illumina sequencing reads from the Tn-seq analysis were deposited in the NCBI Sequence Read Archive (SRA) and released under the accession number SRP150081.

The Tn-seq data, analyses, and pipeline for the Tn-seq analyses are accessible under the DOI number 10.5281/zenodo.2541163 in GitHub as the following link http://doi.org/10.5281/zenodo.2541163.

\section{Field-specific reporting}

Please select the best fit for your research. If you are not sure, read the appropriate sections before making your selection.

$\bigotimes$ Life sciences $\quad \square$ Behavioural \& social sciences $\quad \square$ Ecological, evolutionary \& environmental sciences

For a reference copy of the document with all sections, see nature.com/authors/policies/ReportingSummary-flat.pdf

\section{Life sciences study design}

All studies must disclose on these points even when the disclosure is negative.

Sample size Because for experiments performed in this manuscript, the sample size was not a factor of the analysis, no calculations of sample size were performed. All sample sizes were determined in accordance with published literature relevant to a particular experiment and they were optimal to generate statistically significant results. In general, the experimental analysis was performed in triplicate unless otherwise indicated. GC-MS measurements of carbohydrate composition and LC-MS analysis of GroP were performed twice. X-ray data were collected from single crystals.

Data exclusions No data was excluded from analysis.

Replication All replicates were performed in independent measurements and they were successful.

Tn-seq data analysis including sample diagnostic analyses (PCA, heirarchical clustering) are accessible under the DOI number 10.5281/ zenodo.2541163 in GitHub as the following link http://doi.org/10.5281/zenodo.2541163

Randomization No randomization was applied to this study. Each biochemical assay was designed and performed with controls to avoid biased results. Crystal and glycopolymer samples are not required to be allocated into experimental groups in structural studies, and no animals or human research participants are involved in this study.

Blinding

No human participants or animals were employed in our works, so there was no issue about blinding for group allocation.

\section{Reporting for specific materials, systems and methods}


Materials \& experimental systems

\begin{tabular}{|c|c|}
\hline$n / a$ & Involved in the study \\
\hline & Х Unique biological materials \\
\hline & $\bigotimes$ Antibodies \\
\hline & Eukaryotic cell lines \\
\hline & Palaeontology \\
\hline & Animals and other organisms \\
\hline & Human research participants \\
\hline
\end{tabular}

Methods

$\mathrm{n} / \mathrm{a}$ Involved in the study

X $\square$ chIP-seq

Х $\square$ Flow cytometry

Х $\square$ MRI-based neuroimaging

\section{Unique biological materials}

Policy information about availability of materials

Obtaining unique materials

The bacterial strains and plasmids generated in this study are available upon request from the corresponding authors Natalia Korotkova and Nina van Sorge.

\section{Antibodies}

Antibodies used

Validation
Anti-Streptococcus pyogenes Group A Carbohydrate goat polyclonal antibodies, Abcam, ab9191, lots GR3231184-1 and GR282892-1; dilution 2,500.

The pAb ab9191 were validated previously by Rush et al., 2017 (JBC, 292, 19441-19457) and has been used extensively in GAS field. Further validation information is available on the vendor website: https://www.abcam.com/streptococcus-pyogenesgroup-a-carbohydrate-antibody-ab9191.html 